\title{
On real-time robust model predictive control ${ }^{\text {is }}$
}

\author{
Melanie N. Zeilinger ${ }^{\mathrm{a}, \mathrm{b}, 1}$, Davide M. Raimondo ${ }^{\mathrm{e}}$, Alexander Domahidi ${ }^{\mathrm{d}}$, \\ Manfred Morari ${ }^{\mathrm{d}}$, Colin N. Jones ${ }^{\mathrm{c}}$ \\ a Department of Electrical Engineering and Computer Sciences, UC Berkeley, CA 94720, USA \\ ${ }^{\mathrm{b}}$ Department of Empirical Inference, Max Planck Institute for Intelligent Systems, 72076 Tübingen, Germany \\ ${ }^{\mathrm{c}}$ Laboratoire d'Automatique, École Polytechnique Fédérale de Lausanne (EPFL), 1015 Lausanne, Switzerland \\ d Automatic Control Laboratory, ETH Zurich, Physikstrasse 3, ETL I 28, CH8092 Zurich, Switzerland \\ e Dipartimento di Ingegneria Industriale e dell'Informazione, Università di Pavia, via Ferrata 1, 27100 Pavia, Italy
}

\section{A R T I C L E I N F O}

\section{Article history:}

Received 11 June 2012

Received in revised form 7 May 2013

Accepted 12 October 2013

Available online $\mathrm{xxxx}$

\section{Keywords:}

Real-time model predictive control

Linear systems

Optimal control

\begin{abstract}
A B S T R A C T
High-speed applications impose a hard real-time constraint on the solution of a model predictive control (MPC) problem, which generally prevents the computation of the optimal control input. As a result, in most MPC implementations guarantees on feasibility and stability are sacrificed in order to achieve a realtime setting. In this paper we develop a real-time MPC approach for linear systems that provides these guarantees for arbitrary time constraints, allowing one to trade off computation time vs. performance. Stability is guaranteed by means of a constraint, enforcing that the resulting suboptimal MPC cost is a Lyapunov function. The key is then to guarantee feasibility in real-time, which is achieved by the proposed algorithm through a warm-starting technique in combination with robust MPC design. We address both regulation and tracking of piecewise constant references. As a main contribution of this paper, a new warm-start procedure together with a Lyapunov function for real-time tracking is presented. In addition to providing strong theoretical guarantees, the proposed method can be implemented at high sampling rates. Simulation examples demonstrate the effectiveness of the real-time scheme and show that computation times in the millisecond range can be achieved.
\end{abstract}

(C) 2013 Elsevier Ltd. All rights reserved.

\section{Introduction}

Computation of the optimal model predictive control (MPC) input is generally not practical when controlling high speed systems, which impose a strict real-time constraint on the solution of an MPC problem, i.e. a limit on the computation time that is available to compute the control input. The goal is then to provide a suboptimal control action within the time constraint that still guarantees stability of the closed-loop system and achieves acceptable performance. In this paper, we develop a real-time MPC scheme

\footnotetext{
The research leading to these results has received funding from the European Union Seventh Framework Programme FP7/2007-2013 under grant agreements FP7-ICT-223866 (Feednetback) and FP7-ICT-248940 (EMBOCON). The material in this paper was partially presented at the 48th IEEE Conference on Decision and Control (CDC) and 28th Chinese Control Conference (CCC), December 16-18, 2009, Shanghai, PR China. This paper was recommended for publication in revised form by Associate Editor Yasumasa Fujisaki under the direction of Editor Roberto Tempo.$$
\text { E-mail addresses: mzeilinger@berkeley.edu (M.N. Zeilinger) }
$$

davide.raimondo@unipv.it (D.M. Raimondo), domahidi@control.ee.ethz.ch (A. Domahidi),morari@control.ee.ethz.ch (M. Morari),colin.jones@epfl.ch (C.N. Jones).

1 Tel.: +1 5106435105; fax: +1 5106432356 .
}

that guarantees stability and constraint satisfaction for all realtime constraints and allows for fast online computation. The apriori stability guarantee allows one to trade the performance of the suboptimal controller for lower online computation times.

In online MPC approaches, a constrained optimal control problem is solved at each time instant. Several methods have been proposed in recent years, demonstrating that the computation times can be pushed into a range where online optimization can be used for the control of high-speed systems (Ferreau, Bock, \& Diehl, 2008; Wang \& Boyd, 2010). Significant reduction of the computational complexity can be achieved by exploiting the particular structure and sparsity inherent in the MPC problem (Axehill \& Hansson, 2008; Milman \& Davison, 2008; Wang \& Boyd, 2010; Wright, 1997). In a real-time environment, all available methods for fast online MPC resort to early termination, sacrificing guarantees on either feasibility or stability of the applied control action.

The main contribution of this paper is to show how feasibility and input-to-state stability can be guaranteed in a real-time MPC approach for linear systems under additive disturbances using robust MPC design, a stability enforcing constraint and a warm-start procedure, while allowing for low computation times. While Lyapunov stability and robust MPC theory are well established techniques, we demonstrate how to employ these tools to construct a 
practical high-speed and real-time MPC method with guarantees for regulation, but most importantly also for tracking piecewise constant references, a problem that is faced in many control applications.

The key is to ensure satisfaction of all constraints including the stability constraint in real-time, which is achieved by the presented warm-start procedure. While a feasible and stabilizing warm-start is well-known in the regulation case, a warm-start for tracking with these properties is not directly available. Employing the tracking approach presented in Limon, Alvarado, Alamo, and Camacho (2008), which provides recursive feasibility with respect to state and input constraints, we show that a warm-start strictly satisfying the stability constraint for the tracking MPC problem exists at all iterations and can be generated with negligible computational effort. A Lyapunov function is derived for the tracking approach, which has not been shown in the literature, but is crucial for proving input-to-state stability in the real-time case. Once feasibility is established, the stability constraint ensures that the improved suboptimal solution obtained from the optimization can be safely applied to the system and the performance is improved when more computation time is available.

The proposed robust real-time MPC problem (for tracking) results in a convex quadratically constrained quadratic program (QCQP) with a structure that is different from a standard MPC problem. Implementation details for exploiting this new structure are discussed and computational results based on the efficient implementation techniques introduced in Domahidi, Zgraggen, Zeilinger, Morari, and Jones (2012) are presented, demonstrating that high-speed computation times can be achieved even for realtime tracking. For a 6-dimensional example system, five interiorpoint iterations for the tracking problem were computed in less than $300 \mu$ s with an average performance deterioration of less than $5.6 \%$.

The outline of the paper is as follows: Section 2 introduces the notation and some preliminary results. In Section 3 the challenges of real-time MPC are introduced. Section 4 presents the proposed real-time robust MPC method for regulation and proves inputto-state stability of the closed-loop system under the real-time control law. The results are extended to the more general case of tracking piecewise constant references in Section 5. In Section 6, some implementation details for fast computation are discussed. Finally, Section 7 illustrates the proposed techniques and their advantages using numerical examples.

\section{Notation \& preliminaries}

Let $\mathbb{N}$ denote the set of non-negative integers. A polyhedron is the intersection of a finite number of halfspaces $P=\{x \mid A x \leq b\}$ and a polytope is a bounded polyhedron. If $A \in \mathbb{R}^{m \times n}$ then $A_{i} \in \mathbb{R}^{n}$ is the vector formed by the $i$ th row of $A$. If $b \in \mathbb{R}^{m}$ is a vector, then $b_{i}$ is the $i$ th element of $b$. Given two sets $S_{1}, S_{2} \subseteq \mathbb{R}^{n}$, the Minkowski sum is defined as $S_{1} \oplus S_{2} \triangleq\left\{s_{1}+s_{2} \mid s_{1} \in S_{1}, s_{2} \in S_{2}\right\}$ and the Pontryagin difference as $S_{1} \ominus S_{2} \triangleq\left\{s \mid s+s_{2} \in S_{1}, s_{2} \in S_{2}\right\}=$ $\left\{s \mid s \oplus S_{2} \subseteq S_{1}\right\}$. A weighted 2-norm with $Q \succeq 0$ is denoted by $\|x\|_{Q} \triangleq\left\|Q^{1 / 2} x\right\|_{2}$. Given a sequence $\mathbf{u} \triangleq\left[u_{0}, \ldots, u_{N-1}\right], u_{j}$ denotes the $j$ th element of $\mathbf{u}$; the cardinality of the sequence is inferred from the context. The dependence of a sequence on a parameter is denoted by $\mathbf{u}(x)$, while $u_{j}(x)$ denotes its $j$ th element. A function $\gamma: \mathbb{R}_{\geq 0} \rightarrow \mathbb{R}_{\geq 0}$ is of class $\mathcal{K}$ if it is continuous, strictly increasing and $\gamma(0)=0$ (Vidyasagar, 1993). If in addition $\gamma(s) \rightarrow \infty$ as $s \rightarrow \infty$, then it is of class $\mathcal{K}_{\infty}$. A function $\beta: \mathbb{R}_{\geq 0} \times \mathbb{R}_{\geq 0} \rightarrow \mathbb{R}_{\geq 0}$ is of class $\mathcal{K} \mathcal{L}$ if, for each fixed $t \geq 0, \beta(\cdot, t)$ is of class $\mathcal{K}$, for each fixed $s \geq 0, \beta(s, \cdot)$ is non-increasing and $\beta(s, t) \rightarrow 0$ as $t \rightarrow \infty$ (Vidyasagar, 1993).

Consider the discrete-time uncertain linear system

$x(k+1)=A x(k)+B u(k)+w(k), \quad k \in \mathbb{N}$ that is subject to the following constraints:

$x(k) \in \mathbb{X} \subset \mathbb{R}^{n}, \quad u(k) \in \mathbb{U} \subset \mathbb{R}^{m}$,

where $x(k)$ is the state, $u(k)$ is the control input and $w(k) \in \mathcal{W} \subset$ $\mathbb{R}^{n}$ is a bounded disturbance at the $k^{\prime}$ th sample time. $\mathbb{X}$ and $\mathbb{U}$ are polytopic constraints on the states and inputs that each contain the origin in the interior and $\mathcal{W}$ is a convex and compact disturbance set that contains the origin. When it is convenient, we make use of the lighter notation $x^{+}$to denote the successor state of state $x$ at the next sampling time and $x^{-}$to denote the state at the previous sampling time. The corresponding nominal system is given by

$\bar{x}(k+1)=A \bar{x}(k)+B \bar{u}(k)$.

The solution of the uncertain system controlled by the control law $u(k)=\kappa(x(k))$ at sampling time $k$ for the initial state $x(0)$ and for a sequence of disturbances $\mathbf{w}$ is denoted as $\phi_{\kappa}(k, x(0), \mathbf{w})$.

Assumption 2.1. The pair $(A, B)$ is stabilizable.

The following standard definitions can be found in Blanchini (1999).

Definition 2.2 ((Robust) Positively Invariant Set). A set $S \subseteq \mathbb{R}^{n}$ is a positively invariant (PI) set of system $x(k+1)=f(\bar{x}(k))$, if $f(x(k)) \in S$ for all $x(k) \in S$. A set $S \subseteq \mathbb{R}^{n}$ is a robust positively invariant (RPI) set of system $x(k+1)=f(x(k))+w(k)$, if $f(x(k))+$ $w(k) \in S$ for all $x(k) \in S, w(k) \in \mathcal{W}$.

Definition 2.3 (Regional ISS, Jiang $\mathcal{E}$ Wang, 2001; Sontag $\mathcal{E}$ Wang, 1999). Given an RPI set $\Gamma \subseteq \mathbb{R}^{n}$ containing the origin in its interior, system (1) under the control law $u(k)=\kappa(x(k))$ is Input-toState Stable (ISS) in $\Gamma$ with respect to $w \in \mathcal{W}$, if there exist a $\mathcal{K} \mathcal{L}$-function $\beta$ and a $\mathcal{K}$-function $\gamma$ such that for all initial states $x(0) \in \Gamma$ and for all disturbance sequences $\mathbf{w} \triangleq\left[w_{j}\right]_{j \geq 0}$ with $w_{j} \in W:\left\|\phi_{\kappa}(k, x(0), \mathbf{w})\right\| \leq \beta(\|x(0)\|, k)+\gamma\left(\left\|\mathbf{w}_{[0, k-1]}\right\|\right) \forall k \geq 0$, where $\left\|\mathbf{w}_{[0, k-1]}\right\| \triangleq \max \left\{\left\|w_{j}\right\|, j \in[0, k-1]\right\}$.

Note that the condition for input-to-state stability reduces to that for asymptotic stability if $\mathbf{w}=0$.

Theorem 2.4 (Limon et al., 2009; Rawlings \& Mayne, 2009). Let $\Gamma$ be an RPI set for system (1) under the control law $u(k)=\kappa(x(k))$ and $S \subseteq \Gamma$ be a compact set, both including the origin as an interior point. If there exist a function $V: \mathbb{R}^{n} \rightarrow \mathbb{R}_{+}$, suitable $\mathcal{K}_{\infty}$-class functions $\alpha_{1}, \alpha_{2}, \alpha_{3}$ and a $\mathcal{K}$-class function $\gamma_{2}$ such that

$V(x) \geq \alpha_{1}(\|x\|) \quad \forall x \in \Gamma$
$V(x) \leq \alpha_{2}(\|x\|) \quad \forall x \in S$
$V(A x+B \kappa(x)+w)-V(x) \leq-\alpha_{3}(\|x\|)+\gamma_{2}(\|w\|) \quad \forall x \in \Gamma, w \in W$

$V(\cdot)$ is called an ISS Lyapunov function in $\Gamma$ and the system $x(k+1)=$ $A x(k)+B \kappa(x(k))+w(k)$ is ISS in $\Gamma$ with respect to $w \in \mathcal{W}$.

\section{Problem statement}

Iterative algorithms generally resort to the early termination of the optimization procedure in order to achieve a real-time guarantee. However, stopping a general optimization solver based on an interior-point method early invalidates the theoretical guarantees on recursive feasibility, hence constraint satisfaction, and stability of the classical MPC controller, which are based on optimality (Mayne, Rawlings, Rao, \& Scokaert, 2000). This is true even when the optimization is started from a feasible and stabilizing suboptimal solution. Interior-point methods are, however, required for solving many practical MPC problems, e.g. when using 


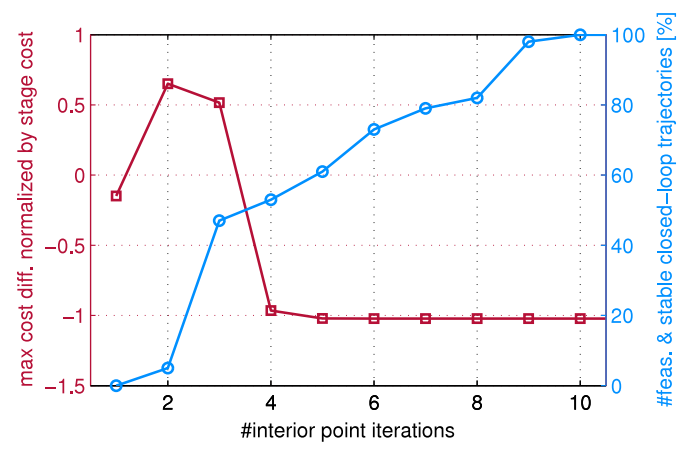

Fig. 1. Percentage of initial states resulting in feasible closed-loop trajectories satisfying the stability condition (out of 100 randomly sampled states) (o) and maximum cost difference in nominal cost between two time steps normalized by stage cost in closed-loop for one initial state ( $\square)$.

quadratic constraints. The loss of the system theoretic properties is due to the following aspects.

An interior-point method does generally not ensure a decrease in the cost to minimize at each iteration of the optimization. This can be easily understood considering a barrier interior-point method, where the cost is augmented by a barrier function. While at each interior-point iteration the augmented cost is decreased, the original cost, which is the MPC cost in our case, may increase; an effect that is particularly prominent during the first few iterations. This is critical for a real-time approach, where often only a small number of iterations can be executed. Even when starting the optimization from a feasible and stabilizing control sequence, a cost decrease can hence not be guaranteed and the MPC cost cannot be employed as a Lyapunov function, as is done in a standard MPC approach.

Feasibility is lost in practice due to model inaccuracies or disturbances rendering an initialization based on the previous time instant infeasible. Since it can generally not be guaranteed that feasibility is recovered by the optimization procedure in a fixed amount of time when starting from an infeasible solution, a feasible initialization is of critical importance in a real-time method.

These issues are demonstrated for a system of six oscillating masses. More details about the example problem are given in Section 7, where the spring constant is here chosen to 1 and there is no damping. A standard nominal MPC problem is solved using a cold-start interior-point method with a given fixed number of iterations and applied in closed-loop to the nominal system in (3). Fig. 1 illustrates the percentage of initial states out of 100 randomly chosen samples that result in closed-loop trajectories not only satisfying all state and input constraints, but also the stability condition, i.e. a decrease in the MPC cost from one time step to the next. Using 5 online iterations, about $40 \%$ of the trajectories are infeasible and/or not guaranteed to be stable and even after 9 interior-point iterations a safe solution cannot be provided for all initial states. In addition, Fig. 1 shows the maximum difference of the MPC cost between two time steps normalized by the stage cost for one particular initial state, i.e. a positive value represents a cost increase and violation of the stability condition. While performing one interior-point iteration still results in a safe solution with guaranteed stability, two or three iterations lead to a loss of this property. Performing more iterations can hence even deteriorate the system theoretic properties, demonstrating the need for an MPC method with real-time feasibility and stability guarantees.

In this work, feasibility with respect to the state and input constraints despite disturbances is recovered by using a tube based robust MPC scheme (Mayne, Seron, \& Rakovic, 2005). The main challenge is to guarantee real-time stability of the closedloop system, which is achieved by introducing what we term a Lyapunov constraint, explicitly enforcing that the real-time MPC cost is an ISS Lyapunov function. A warm-start procedure is introduced in order to ensure feasibility of the Lyapunov constraint in real-time.

In order to achieve tracking of a desired sequence of steadystates rather than regulation around the origin or to a particular steady state, it is standard practice to modify the MPC problem by means of a change of variables (see e.g. Maciejowski (2000), Rawlings and Mayne (2009)). This problem formulation does, however, not provide recursive feasibility. In this paper, a realtime MPC method for tracking is developed based on the tracking approach in Limon et al. (2008), and a new warm-start is proposed providing feasibility and stability in real-time for all time constraints.

\section{Real-time robust MPC with guarantees}

Consider the discrete-time uncertain system in (1). The realtime MPC procedure proposed in this paper is based on both a modified MPC problem formulation as well as a real-time algorithm to guarantee robust invariance and input-to-state stability of the closed loop system. In the following, a control law is called $\tau$-real time ( $\tau$-RT) if it is computed in $\tau$ seconds.

We propose the following real-time robust MPC problem in order to realize a $\tau$-RT control law.

Problem. $\mathbb{P}_{N}^{\tau}(x)$ (Real-time robust MPC problem)

$$
\begin{aligned}
V_{N}(\overline{\mathbf{x}}, \overline{\mathbf{u}}) \triangleq & \sum_{i=0}^{N-1} l\left(\bar{x}_{i}, \bar{u}_{i}\right)+V_{f}\left(\bar{x}_{N}\right) \\
\min _{\overline{\mathbf{x}}, \overline{\mathbf{u}}} & V_{N}(\overline{\mathbf{x}}, \overline{\mathbf{u}})+V_{f}\left(x-\bar{x}_{0}\right) \\
\text { s.t. } \quad & \bar{x}_{i+1}=A \bar{x}_{i}+B \bar{u}_{i}, \quad i=0, \ldots, N-1, \\
& \left(\bar{x}_{i}, \bar{u}_{i}\right) \in \overline{\mathbb{X}} \times \overline{\mathbb{U}}, \quad i=0, \ldots, N-1, \\
& \bar{x}_{N} \in \bar{X}_{f}, \\
& V_{N}(\overline{\mathbf{x}}, \overline{\mathbf{u}})+V_{f}\left(x_{\text {nom }}-\bar{x}_{0}\right) \leq \Pi_{\mathrm{prev}}, \\
& x \in \bar{x}_{0} \oplus \mathcal{Z}_{W} .
\end{aligned}
$$

A quadratic stage cost and terminal penalty function are chosen, i.e. $l(x, u) \triangleq\|x\|_{Q}^{2}+\|u\|_{R}^{2}, V_{f}(x) \triangleq\|x\|_{P}^{2}$, where $Q, R$ and $P$ are positive definite matrices, and $\bar{X}_{f}$ is an invariant terminal target set. The constants $x_{\text {nom }}$ and $\Pi_{\text {prev }}$ are defined in Definition 4.2 below.

Problem $\mathbb{P}_{N}^{\tau}(x)$ differs from a standard nominal MPC problem in two main aspects: the use of the tube based robust MPC problem setup in Mayne et al. (2005) to provide recursive feasibility of the state and input constraints and a Lyapunov constraint enforcing input-to-state stability at all iterations following the ideas in Scokaert, Mayne, and Rawlings (1999).

Robust MPC design: the robust MPC formulation reduces the control to the tube centers $\overline{\mathbf{x}}$, which are steered to the origin by choosing a sequence of control inputs $\overline{\mathbf{u}}$ and the first tube center $\bar{x}_{0}$. Constraints (5d) and $(5 \mathrm{~g})$ provide robustness with respect to the additive disturbance $w$ in (1), where $\mathcal{Z}_{w}$ is an RPI set for system (1) under the control law $u(k)=K x(k)$ with $w(k) \in W \forall k \in \mathbb{N}$. $\overline{\mathbb{X}}=\mathbb{X} \ominus \mathcal{Z}_{w}, \overline{\mathbb{U}}=\mathbb{U} \ominus K \mathcal{Z}_{w}$ are tightened constraints on the states and inputs ensuring constraint satisfaction of the uncertain system in (1) despite the disturbance $w$. Note that the first tube center $\bar{x}_{0}$ is an optimization variable satisfying $(5 \mathrm{~g})$ that may differ from the current state measurement $x$. Compared to Mayne et al. (2005) we propose to augment the cost in (5b) by the term $V_{f}\left(x-\bar{x}_{0}\right)$, introducing a tradeoff between the amount of control action used for counteracting the disturbance and the effort for controlling the tube centers to the origin. The main advantage of 
the augmented cost is that it provides an ISS Lyapunov function for the closed-loop system under the real-time robust MPC control law (see Theorem 4.7), whereas the approach in Mayne et al. (2005) does not. Note that the availability of an ISS Lyapunov function is also beneficial in an optimal setup by characterizing the closed-loop behavior as a function of the disturbance realization. Apart from the additional cost term, we directly employ the robust formulation and refer to Mayne et al. (2005) for more details and an illustrative example of the approach.

Lyapunov constraint: the constraint (5f) ensures that the suboptimal cost achieved after $\tau$ seconds satisfies the Lyapunov decrease condition, which is essential in order for the MPC cost to provide an ISS Lyapunov function when using an interiorpoint method. It explicitly enforces that the MPC cost (5b) at $x(k)$ decreases with respect to the cost at the last sample time if the system satisfies the nominal dynamics and thereby recovers the stability properties of the optimal robust MPC approach in a realtime setting. The Lyapunov decrease constraint (5f) represents a convex quadratic constraint on the optimization variables.

Instead of solving problem $\mathbb{P}_{N}^{\tau}(x)$ to optimality, the optimization is executed at $x(k)$ for no more than $\tau$ seconds before returning the variables $\overline{\mathbf{u}}^{\tau}(x(k))$ and $\overline{\mathbf{x}}^{\tau}(x(k))$. The robust $\tau$-RT control law is then given in a receding horizon control fashion by

$\kappa^{\tau}(x(k))=\bar{u}_{0}^{\tau}(x(k))+K\left(x(k)-\bar{x}_{0}^{\tau}(x(k))\right)$.

Definition 4.1 ( $\tau$-RT Cost). We define $V^{\tau}(x)=V_{N}\left(\overline{\mathbf{x}}^{\tau}(x), \overline{\mathbf{u}}^{\tau}(x)\right)+$ $V_{f}\left(x-\bar{x}_{0}^{\tau}(x)\right)$ to be the cost of the suboptimal solution obtained after solving Problem $\mathbb{P}_{N}^{\tau}(x)$ for $\tau$ seconds.

Definition 4.2 (Lyapunov Constraint). For each $x(k)$, we take

$x_{\text {nom }}=A x(k-1)+B \kappa^{\tau}(x(k-1))$

to be the state that would have been obtained in the absence of disturbances and

$\Pi_{\text {prev }}=V^{\tau}(x(k-1))-\frac{1}{2} \epsilon\|x(k-1)\|_{Q}^{2}$,

where $x(k-1)$ denotes the state at the previous, i.e. the $(k-1)$ 'th, sample time and $\epsilon \in(0,1)$ is a user-specified constant. Note that $\epsilon<1$ is required for the use of an interior point method, since the applied warm-start introduced in Algorithm 1 satisfies (7) with $\epsilon=1$ (see Lemma 4.6). $\epsilon>0$ is required in order to prove stability, as will be shown in Theorem 4.7 .

Problem $\mathbb{P}_{\underline{N}}^{\tau}(x)$ implicitly defines the set of feasible control sequences $\bar{u}_{N}\left(\bar{x}_{0}\right) \triangleq\{\overline{\mathbf{u}} \mid \exists \overline{\mathbf{x}}$ s.t. (5c) - (5f) hold $\}$, feasible initial tube centers $\left.\bar{X}_{0}(x) \triangleq\left\{\bar{x}_{0}\right\rfloor(5 \mathrm{~g})\right\}$ and feasible initial states $\mathcal{X}_{N} \triangleq$ $\left\{x \mid \exists \bar{x}_{0} \in \bar{X}_{0}(x)\right.$ s.t. $\left.\bar{u}_{N}\left(\bar{x}_{0}\right) \neq \emptyset\right\}$. We make the following standard assumption.

Assumption 4.3. $V_{f}(\cdot)$ is a Lyapunov function in $\bar{X}_{f}$ and $\bar{X}_{f}$ is a PI set for system (3) under the control law $\kappa_{f}(x)=K x$, given by the following conditions:

A1: $\bar{x}_{f} \subseteq \overline{\mathbb{X}},(A+B K) \bar{x}_{f} \subseteq \bar{x}_{f}, K \bar{x}_{f} \subseteq \overline{\mathbb{U}}$

A2: $V_{f}((A+B K) x)-V_{f}(x) \leq-l(x, K x) \forall x \in \bar{x}_{f}$.

Assumption 4.4. It is assumed that $\mathcal{Z}_{w} \subseteq \bar{X}_{f}$.

Note that in the considered case of a linear system model, a terminal control law and terminal cost satisfying Assumption 4.3 are given by the infinite horizon LQR control law and cost. The terminal set can then be chosen as a level set of the terminal cost of suitable size. Assumption 4.4 can be relaxed, if $V_{f}(\cdot)$ is a Lyapunov function in $\mathcal{Z}_{w}$, i.e. if condition A2 in Assumption 4.3 is satisfied for all $x \in \mathbb{Z}_{W}$.
Remark 4.5. The real-time robust MPC problem $\mathbb{P}_{N}^{\tau}(x(k))$, the real-time solution $\bar{x}_{0}^{\tau}(x(k)), \overline{\mathbf{u}}^{\tau}(x(k))$, the corresponding $\tau$-RT cost $V^{\tau}(x(k))$ as well as the resulting robust $\tau$-RT control law $\kappa^{\tau}(x(k))$ in (6) are not only a function of the current state, but also of the previous state, the real-time solution $\overline{\mathbf{u}}^{\tau}(x(k-1))$ and $\bar{x}_{0}^{\tau}(x(k-$ 1)) computed at the previous time step as well as the available computation time $\tau$, which are, however, given constants at the time of computation. We omit this dependence for the ease of notation, but denote the dependence on the solution from the previous time step and the computation time by the index $\tau$.

Any feasible solution to Problem $\mathbb{P}_{N}^{\tau}(x)$ will satisfy the Lyapunov decrease condition, the main property to ensure that the $\tau$-RT cost is an ISS Lyapunov function, and can be used to construct a control law with real-time stability guarantees. While input-tostate stability therefore follows from the addition of the Lyapunov constraint, the challenge has moved to ensuring that a feasible solution can be found in a pre-specified amount of time. As discussed before, satisfaction of constraints in real-time can only be guaranteed if an initial feasible solution is available. The key is hence to provide a warm-start achieving some epsilon progress without adding significant computational effort. While in the regulation case a standard warm-start employing the real-time solution at the previous time step directly offers this property (Lemma 4.6), this represents the main challenge in the extension to reference tracking in Section 5.

We propose the real-time Algorithm 1, which implements a warm-start procedure to initialize a primal feasible optimization routine that is terminated after an arbitrary computation time $\tau$. Since Problem $\mathbb{P}_{N}^{\tau}(x)$ contains quadratic constraints, interiorpoints methods provide suitable and efficient optimizers for Step 4, e.g. a primal barrier method or a primal-dual method with primal feasible iterates (Boyd \& Vandenberghe, 2004; Nocedal \& Wright, 2006).

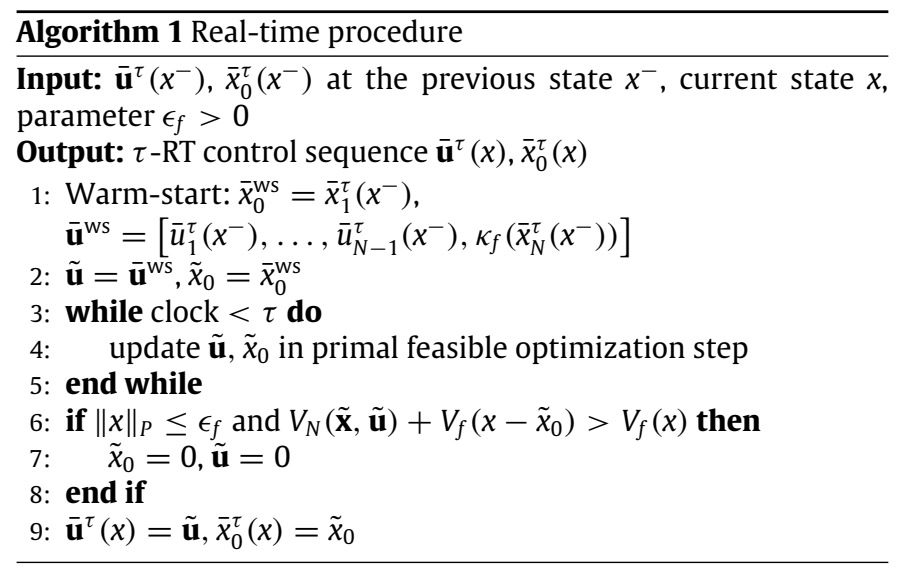

Algorithm 1 differs from a standard approach in the following main aspect.

Upper bound on Lyapunov function: steps 6-8 ensure that the real-time cost can be upper bounded by a $\mathcal{K}_{\infty}$-class function of the state in a set that includes the origin in its interior. The use of $V_{f}(x)$ as a local upper bound is a standard technique in the stability analysis of MPC, see e.g. Magni, Raimondo, and Scattolini (2006), but is not automatically satisfied by the employed Lyapunov function. The real-time solution obtained from Problem $\mathbb{P}_{N}^{\tau}(x)$ is only guaranteed to provide a lower cost compared to the previous sampling time, but may result in a bigger cost than the auxiliary control law even for an initial state close to the origin. A local upper bound is therefore enforced, here we also choose $V_{f}(x)$, since it represents the cost for using $\tilde{x}_{0}=0, \tilde{\mathbf{u}}=0$. The bound holds in the set $\varepsilon_{f} \triangleq\left\{x \mid\|x\|_{P} \leq \epsilon_{f}\right\}$, which can be taken as any subset 
of $\mathfrak{Z}_{w}$ containing $\tilde{x}_{0}=0, \tilde{\mathbf{u}}=0$ as a feasible point. The upper bound together with the Lyapunov decrease constraint (5f) provide that the MPC cost is an ISS Lyapunov function (see Theorem 4.7). While Steps 6-8 are therefore mainly motivated from theoretical considerations, they are beneficial in a real-time environment, since the auxiliary control law is used if it provides a lower cost than the solution obtained in the real-time optimization.

The following lemma shows that the warm-start proposed in Algorithm 1 is in fact feasible for $\mathbb{P}_{N}^{\tau}(x)$ with respect to all state and input constraints as well as the Lyapunov decrease constraint. The proof can be found in the appendix.

Lemma 4.6 (Feasibility of the Warm-Start). Let $\overline{\mathbf{u}}^{\tau}(x(k-1))$, $\bar{x}_{0}^{\tau}(x(k-1))$ be a feasible control sequence and tube center for Problem $\mathbb{P}_{N}^{\tau}(x(k-1))$. The warm-start solution provided by Algorithm 1 is feasible for $\mathbb{P}_{N}^{\tau}(x(k))$, where $x(k) \in A x(k-1)+$ $B \kappa^{\tau}(x(k-1)) \oplus$ w, i.e. $\bar{x}_{0}^{w s} \in \bar{X}_{0}(x(k)), \overline{\mathbf{u}}^{w s} \in \bar{u}_{N}\left(\bar{x}_{0}^{w s}\right)$.

Starting from a feasible warm-start solution, feasibility is maintained by using a primal feasible optimization method in Algorithm 1, providing recursive feasibility of the closed-loop system under the proposed $\tau$-RT control law. Due to the Lyapunov constraint (5f), feasibility of $\mathbb{P}_{N}^{\tau}(x)$ implies input-to-state stability, which allows us to state the main result of this section and prove ISS of the closed-loop system under the robust $\tau$-RT control law $\kappa^{\tau}(x)$ in (6). Note that in the considered case stability cannot be achieved by the approach described in Lazar, Roset, Heemels, Nijmeijer, and van den Bosch (2008), where a constraint on the Lyapunov decrease is only introduced in the first step, since the solutions are not recursively feasible.

Theorem 4.7 (Stability Under $\kappa^{\tau}(x)$ ). Consider Problem $\mathbb{P}_{N}^{\tau}(x)$ fulfilling Assumption 4.3. The closed-loop system (1) under the $\tau$-RT control law in (6) that is obtained from Algorithm 1 is ISS w.r.t. $w(k) \in \mathcal{W}$ with region of attraction $\mathcal{X}_{N}$ for all $\tau \geq 0$.

Proof. We will show that $V^{\tau}(x)$ provides an ISS Lyapunov function. Assumption 4.3 provides that $\|\cdot\|_{Q}^{2} \leq\|\cdot\|_{P}^{2}$, since $\|x\|_{Q}^{2} \leq\|x\|_{P}^{2}-$ $\left\|x^{+}\right\|_{P}^{2}$. Using convexity of $\|\cdot\|_{Q}^{2}$, it can be shown that there exists a $\mathcal{K}_{\infty}$-class function $\underline{\alpha}(\cdot)$ such that $V^{\tau}(x) \geq\left\|\bar{x}_{0}^{\tau}(x)\right\|_{Q}^{2}+\left\|x-\bar{x}_{0}^{\tau}(x)\right\|_{Q}^{2}$ $\geq \frac{1}{2}\|x\|_{Q}^{2} \geq \underline{\alpha}(\|x\|) \forall x \in \mathcal{X}_{N}$. If $\|x\|_{P} \leq \epsilon_{f}$ and Steps 6-8 are not applied: $V^{\bar{\tau}}(\bar{x}) \leq V_{f}(x)$ by the condition in Step 6. If Steps 6-8 are applied: $V^{\tau}(x)=V_{N}(0,0)+V_{f}(x)=V_{f}(x)$. Hence there exists a $\mathcal{K}_{\infty}$-class function $\bar{\alpha}(\cdot)$ such that $V^{\tau}(x) \leq V_{f}(x) \leq \bar{\alpha}(\|x\|)$ $\forall x$ s.t. $\|x\|_{P} \leq \epsilon_{f}$. Note that in order to show ISS using Theorem 2.4, it is sufficient to ensure the upper bound on the Lyapunov function in a neighborhood of the origin, since this bound can be extended to an upper bound on any compact set, see e.g. Proposition 2.18 in Rawlings and Mayne (2009). The last property to show is the Lyapunov decrease. Let $\tilde{\mathbf{u}}(x), \tilde{\mathbf{x}}(x)$ denote the solution that is returned by the optimization in Step 5 . Since it is feasible for $\mathbb{P}_{N}^{\tau}(x)$ by feasibility of the warm-start shown in Lemma 4.6 and the use of a primal feasible optimization routine, it follows from the Lyapunov constraint (5f) that $V_{N}(\tilde{\mathbf{x}}(x), \tilde{\mathbf{u}}(x))+V_{f}\left(x_{\text {nom }}-\tilde{x}_{0}(x)\right)-$ $V^{\tau}\left(x^{-}\right) \leq-\frac{1}{2} \epsilon\left\|x^{-}\right\|_{Q}^{2}$. Recalling that $x=A x^{-}+B \kappa^{\tau}\left(x^{-}\right)+w$, this implies

$$
\begin{aligned}
& V_{N}(\tilde{\mathbf{x}}(x), \tilde{\mathbf{u}}(x))+V_{f}\left(x-\tilde{x}_{0}(x)\right) \\
& \leq V^{\tau}\left(x^{-}\right)-\frac{1}{2} \epsilon\left\|x^{-}\right\|_{Q}^{2}+\left|V_{f}\left(x-\tilde{x}_{0}(x)\right)-V_{f}\left(x_{\text {nom }}-\tilde{x}_{0}(x)\right)\right|(8 \mathrm{~b}) \\
& \leq V^{\tau}\left(x^{-}\right)-\beta\left(\left\|x^{-}\right\|\right)+\gamma(\|w\|) \quad \forall x \in X_{N}
\end{aligned}
$$

where the last step follows from continuity of $V_{f}(x), \gamma(\cdot)$ is a suitable $\mathcal{K}$-class function and $\beta(\cdot)$ a $\mathcal{K}_{\infty}$-class function. If Steps 6-8 are not applied, then $\overline{\mathbf{x}}^{\tau}(x)=\tilde{\mathbf{x}}(x)$ and $\overline{\mathbf{u}}^{\tau}(x)=\tilde{\mathbf{u}}(x)$. If Steps 6-8 are applied, then $\overline{\mathbf{x}}^{\tau}(x)=0$ and $\overline{\mathbf{u}}^{\tau}(x)=0$ and it follows that $V^{\tau}(x)=V_{f}(x) \leq V_{N}(\tilde{\mathbf{x}}(x), \tilde{\mathbf{u}}(x))+V_{f}\left(x-\tilde{x}_{0}(x)\right)$ since the conditions in Step 6 are fulfilled. In all cases we therefore obtain

$V^{\tau}(x) \leq V_{N}(\tilde{\mathbf{x}}(x), \tilde{\mathbf{u}}(x))+V_{f}\left(x-\tilde{x}_{0}(x)\right)$,

which together with (8) shows that $V^{\tau}(x)$ is an ISS Lyapunov function.

Using the robustified problem formulation in $\mathbb{P}_{N}^{\tau}(x)$ with the Lyapunov constraint (5f) together with Algorithm 1, stability of the uncertain system (1) can therefore be guaranteed in a real-time MPC implementation, where the optimization solving $\mathbb{P}_{N}^{\tau}(x)$ is stopped after the available time $\tau$.

Remark 4.8. $\tau$ can be arbitrarily time-varying, which makes the presented approach suitable for operation in a wide range of standard multi-tasking real-time computational platforms.

Remark 4.9. Once a feasible warm-start satisfying the Lyapunov decrease condition is available, a stabilizing strategy could also be constructed without the Lyapunov constraint by resorting to the warm-start whenever the suboptimal solution violates the stability condition. However, if the real-time constraint only allows for a few iterations, the solution is likely to violate the stability condition and the system would run open-loop by applying the warm-start. The Lyapunov constraint, which can be added at no significant computational cost, allows the system to always benefit from performance improvements provided by the optimization.

Remark 4.10. Due to the feasibility guarantee provided by the robust MPC framework, the feasible set $\mathcal{X}_{N}$ is an invariant set for the closed-loop system under the control law that would be obtained from directly applying Algorithm 1 to Problem $\mathbb{P}_{N}^{\tau}(x)$ without the Lyapunov constraint (5f).

Remark 4.11. The use of $\bar{x}_{0}^{\tau}(x)=0, \overline{\mathbf{u}}^{\tau}(x)=0$ results in the control law $\kappa^{\tau}(x)=K x$. By using the auxiliary control law in a neighborhood $\varepsilon_{f}$ of the origin (if the cost cannot be upper bounded by $V_{f}(x)$ ), Algorithm 1 is similar to a dual mode strategy. The difference is that the control strategy does not switch to this control law once the state is inside this set, since $\varepsilon_{f}$ is not robustly invariant. While the existence of a $\mathcal{K}_{\infty}$-class function of the state that upper bounds the suboptimal cost in a neighborhood of the origin is often assumed in suboptimal or real-time methods (e.g. Lazar and Heemels (2009), Scokaert et al. (1999)), Algorithm 1 provides a constructive procedure to satisfy this condition.

Remark 4.12. The re-optimization of the first tube center at every time step introduces additional feedback to the disturbance. A feasible and stable controller could, however, also be obtained by keeping the initial tube center fixed and only optimizing over the sequence of tube centers from $\bar{x}_{1}$ to $\bar{x}_{N}$.

Remark 4.13. The crucial property of recursive feasibility is guaranteed by all available robust MPC methods (see e.g. Bemporad and Morari (1999), Limon et al. (2009), Mayne et al. (2000), Rawlings and Mayne (2009)), any of which could be used to derive a realtime MPC controller for the uncertain system (1). In order to allow for fast computation we use the tube based robust MPC approach for linear systems described in Mayne et al. (2005) in this work.

After establishing feasibility and stability for the $\tau$-RT control law in the regulation case, the following section extends the presented results to the more general case of robust tracking of piecewise constant reference signals. 


\section{Real-time robust MPC for tracking of piecewise constant references}

Consider the task of tracking a piecewise constant sequence of steady-states by steering the system state $x$ to the target steadystate $x_{r}$. A target input $u_{r}$ is associated with every target steadystate $x_{r}$ fulfilling the steady-state condition $x_{r}=A x_{r}+B u_{r}$. The state and input constraints limit the set of feasible steady-states to $\left(x_{r}, u_{r}\right) \in \bar{\Theta}$, where $\bar{\Theta} \triangleq\left\{\left(x_{r}, u_{r}\right) \mid x_{r} \in \overline{\mathbb{X}}, u_{r} \in \overline{\mathbb{U}},(A-I) x_{r}+\right.$ $\left.B u_{r}=0\right\}$.

Remark 5.1. If tracking of an output signal is required, one can translate the output reference $y_{r}$ into a state and input reference $\left(x_{r}, u_{r}\right)$ using the following relation: $\left[\begin{array}{cc}A-I & B \\ C & D\end{array}\right]\left[\begin{array}{l}x_{r} \\ u_{r}\end{array}\right]=\left[\begin{array}{c}0 \\ y_{r}\end{array}\right]$, where $y=C x+D u$ is the output model. Note that a non-trivial solution exists due to Assumption 2.1.

While recursive feasibility of the state and input constraints in the regulation case can be recovered by means of robust MPC design, this is not sufficient when using a standard tracking scheme reformulating the MPC problem by means of a change of variables, since reference changes may render the MPC problem infeasible. In addition, no warm-start is available satisfying the state and input constraints after a reference change due to the fact that the terminal constraint depends on the current reference.

In order to extend the real-time method to reference tracking, we propose a problem formulation based on the tracking approach presented in Limon et al. (2008), which was included in a tube based robust MPC framework in Limon, Alvarado, Alamo, Fiacchini, and Camacho (2010). An artificial reference is introduced into the optimization problem, which may deviate from the real reference if the latter is not a feasible target from the current state. The artificial steady-state and the control sequence are computed by solving a single optimization problem, which provides not only recursive feasibility with respect to state and input constraints but will also allow us to permit a hard real-time guarantee and is the reason for using this somewhat unusual tracking formulation.

A Lyapunov constraint for tracking is again introduced in order to ensure the Lyapunov decrease property for arbitrary real-time constraints. The main challenge in the tracking case is now given by the generation of a warm-start satisfying the Lyapunov constraint, required to guarantee real-time feasibility and stability. In contrast to the regulation case discussed in Section 4, such a warm-start is not readily available for the tracking problem, which will be discussed in more detail in the following. We show that a warmstart strictly satisfying the Lyapunov constraint for the tracking MPC problem based on Limon et al. (2008) exists at all iterations and how it can be generated by a modified warm-start procedure.

We first introduce the real-time robust MPC problem for reference tracking $\mathbb{P}_{N}^{\tau, t r}(x)$ and then present the modified real-time procedure.

Problem. $\mathbb{P}_{N}^{\tau, t r}(x)$ (Real-time robust MPC for reference tracking)

$$
\begin{aligned}
& V_{N}^{t r}\left(\overline{\mathbf{x}}, \overline{\mathbf{u}}, \bar{x}_{s}, \bar{u}_{s}\right) \triangleq \sum_{i=0}^{N-1} l\left(\bar{x}_{i}-\bar{x}_{s}, \bar{u}_{i}-\bar{u}_{s}\right) \\
& +V_{f}\left(\bar{x}_{N}-\bar{x}_{s}\right)+V_{o}\left(\bar{x}_{s}-x_{r}, \bar{u}_{s}-u_{r}\right) \\
& \min _{\overline{\mathbf{x}}, \overline{\mathbf{u}}, \bar{x}_{s}, \bar{u}_{s}} V_{N}^{\text {tr }}\left(\overline{\mathbf{x}}, \overline{\mathbf{u}}, \bar{x}_{s}, \bar{u}_{s}\right)+V_{f}\left(x-\bar{x}_{0}\right) \\
& \text { s.t. (5c), (5d), (5g), } \\
& \left(\bar{x}_{s}, \bar{u}_{s}\right) \in \bar{\Theta} \text {, } \\
& \bar{x}_{N} \in \bar{X}_{f}^{t r}\left(\bar{x}_{s}, \bar{u}_{s}\right) \text {, } \\
& V_{N}^{t r}\left(\overline{\mathbf{x}}, \overline{\mathbf{u}}, \bar{x}_{s}, \bar{u}_{s}\right)+V_{f}\left(x_{\text {nom }}^{t r}-\bar{x}_{0}\right) \leq \Pi_{\text {prev }}^{t r}
\end{aligned}
$$

where $\left(\bar{x}_{s}, \bar{u}_{s}\right)$ denotes the artificial steady-state, $\left(x_{r}, u_{r}\right)$ is the desired steady-state and $V_{o}\left(\bar{x}_{s}-x_{r}, \bar{u}_{s}-u_{r}\right) \triangleq\left\|\bar{x}_{s}-x_{r}\right\|_{T_{x}}^{2}+$ $\left\|\bar{u}_{s}-u_{r}\right\|_{T_{u}}^{2}$ is the tracking offset cost, where $T_{x}$ and $T_{u}$ are positive definite matrices. $\bar{X}_{f}^{\text {tr }}\left(\bar{x}_{s}, \bar{u}_{s}\right)$ is an invariant terminal target set for tracking. The constants $x_{\text {nom }}^{t r}$ and $\Pi_{\text {prev }}^{\text {tr }}$ are defined in Definition 5.2 below. For simplicity of notation, the dependence of $\mathbb{P}_{N}^{\tau, t r}(x)$ and $V_{N}^{\operatorname{tr}}\left(\overline{\mathbf{x}}, \overline{\mathbf{u}}, \bar{x}_{s}, \bar{u}_{s}\right)$ on the desired reference $\left(x_{r}, u_{r}\right)$ is omitted.

In addition to the robust MPC problem setup and the Lyapunov constraint for tracking, the real-time robust MPC problem for reference tracking $\mathbb{P}_{N}^{\tau, t r}(x)$ introduces the following components resulting from the tracking approach (Limon et al., 2008):

- an artificial steady state and input $\left(\bar{x}_{s}, \bar{u}_{s}\right)$, where the cost penalizes the deviation of the states and inputs from the artificial instead of the real reference. A penalty accounting for the offset between the artificial and the real reference is added to the cost and ensures convergence to the desired steady-state $\left(x_{r}, u_{r}\right)$;

- a terminal weight on the deviation between the terminal state and artificial reference as well as an extended terminal constraint on the terminal state and the artificial reference provide stability of the optimal MPC controller.

More details on the tracking formulation, the terminal set for tracking, and an illustrative example can be found in Limon et al. (2008), for the combination with the robust formulation see Limon et al. (2010) or also Zeilinger (2011). The role of the artificial reference can also be observed in the tracking example in Section 7.2.

The optimization solving Problem $\mathbb{P}_{N}^{\tau, t r}(x(k))$ at time step $k$ is again executed for $\tau$ seconds, returning the variables $\overline{\mathbf{u}}^{\tau, \operatorname{tr}}(x(k))$, $\bar{x}_{0}^{\tau, \operatorname{tr}}(x(k)), \bar{x}_{s}^{\tau, \operatorname{tr}}(x(k)), \bar{u}_{\mathrm{s}}^{\tau, \operatorname{tr}}(x(k)) . V^{\tau, \operatorname{tr}}(x(k))$ denotes the corresponding $\tau$-RT cost for tracking. The robust $\tau$-RT control law for tracking is then given in a receding horizon control fashion by:

$\kappa^{\tau, \operatorname{tr}}(x(k))=\bar{u}_{0}^{\tau, \operatorname{tr}}(x(k))+K\left(x(k)-\bar{x}_{0}^{\tau, \operatorname{tr}}(x(k))\right)$.

Definition 5.2 (Lyapunov Constraint for Tracking). For each $x(k)$, we again take

$x_{\mathrm{nom}}^{\text {tr }}=A x(k-1)+B \kappa^{\tau, \operatorname{tr}}(x(k-1))$,

$\Pi_{\mathrm{prev}}^{\text {tr }}=V^{\tau, \operatorname{tr}}(x(k-1))-\frac{1}{2} \epsilon_{k}\left\|(x(k-1))-x_{r}\right\|_{Q}^{2}$,

where $\epsilon_{k} \in(0,1)$ is a positive constant that is chosen at each sampling time such that the warm-start solution is strictly feasible, see Algorithm 2. The existence of such a constant at all times will be shown in Theorem 5.7.

Problem $\mathbb{P}_{N}^{\tau, \operatorname{tr}}(x)$ implicitly defines the set of feasible control sequences $\bar{u}_{N}^{\operatorname{tr}}\left(\bar{x}_{0}, \bar{x}_{s}, \bar{u}_{s}\right) \triangleq\{\overline{\mathbf{u}} \mid \exists \overline{\mathbf{x}}$ s.t. (5c), (5d), (9e), (9f) hold $\}$, and feasible initial states $\mathcal{X}_{N}^{t r} \triangleq\left\{x \mid \exists \bar{x}_{0} \in \bar{X}_{0}(x),\left(\bar{x}_{s}, \bar{u}_{s}\right) \in\right.$ $\bar{\Theta}$ s.t. $\left.\bar{u}_{N}^{t r}\left(\bar{x}_{0}, \bar{x}_{s}, \bar{u}_{s}\right) \neq \emptyset\right\}$.

Assumption 5.3. For a given $\left(\bar{x}_{s}, \bar{u}_{s}\right) \in \bar{\Theta}, V_{f}\left(x-\bar{x}_{s}\right)$ is a Lyapunov function in $\bar{\chi}_{f}^{\text {tr }}\left(\bar{x}_{s}, \bar{u}_{s}\right)$ satisfying Assumption 4.3 and $\bar{X}_{f}^{\text {tr }}\left(\bar{x}_{s}, \bar{u}_{s}\right)$ is a PI set for the nominal system (3) under the local control law for tracking $\kappa_{f}^{\text {tr }}(x)=K\left(x-\bar{x}_{s}\right)+\bar{u}_{s}$, which can be stated as the following condition.

A3: $\bar{X}_{f}^{\operatorname{tr}}\left(\bar{x}_{s}, \bar{u}_{s}\right) \subseteq \mathbb{X}, A x+B \kappa_{f}^{t r}(x) \in \bar{X}_{f}^{t r}\left(\bar{x}_{s}, \bar{u}_{s}\right)$, $\kappa_{f}^{\text {tr }}(x) \in \mathbb{U} \forall x \in \bar{X}_{f}^{t r}\left(\bar{x}_{s}, \bar{u}_{s}\right)$.

It is further assumed that $\mathcal{Z}_{w} \subseteq \bar{\chi}_{f}^{t r}\left(\bar{x}_{s}, \bar{u}_{s}\right) \forall\left(\bar{x}_{s}, \bar{u}_{s}\right) \in \bar{\Theta}$.

The tracking formulation in $\mathbb{P}_{N}^{\tau, \operatorname{tr}}(x)$ is designed to regulate the system state to the artificial steady-state $\left(\bar{x}_{s}, \bar{u}_{s}\right)$, which is simultaneously steered to the target steady-state. Recursive feasibility with respect to the state and input constraints is guaranteed since 
the shifted sequence together with the artificial steady-state from the last sampling time satisfies the constraints (without the Lyapunov constraint) at the current time instant (Limon et al., 2008). Convergence of this scheme to $\left(x_{r}, u_{r}\right)$ using the optimal control law was shown in Limon et al. (2008), Limon et al. (2010) using the fact that $\bar{x}_{s} \neq x_{r}$ cannot be the optimal solution. However, the shifted sequence and artificial steady-state from the last sampling time do not provide a feasible warm-start for the Lyapunov constraint for some strictly positive $\epsilon_{k}$. This is due to the fact that simply shifting the control sequence and applying the local control law will cause the system to converge to the artificial instead of the real reference and the cost decrease will tend to zero although the system has not converged to the desired reference yet.

We therefore propose a new warm-start procedure for tracking in Algorithm 2, which combined with Algorithm 1 enables realtime tracking.

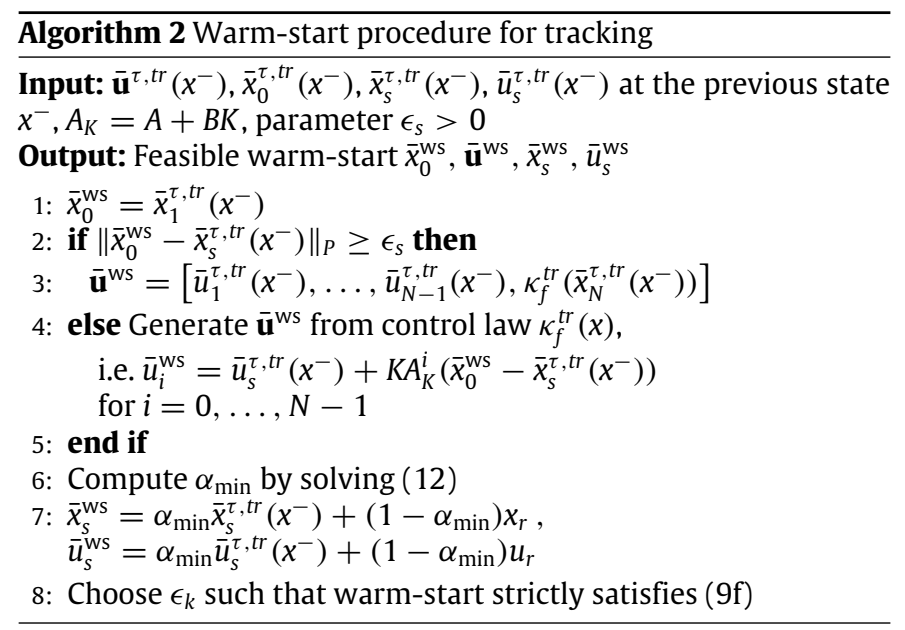

Algorithm 2 introduces the following modifications:

Warm-start input sequence (Steps 2-5): a warm-start sequence is generated either from the shifted initial solution together with an auxiliary control law (Step 3), which was shown to be feasible in Limon et al. (2008), Limon et al. (2010), or, if the initial tube center is close to the artificial steady-state, by applying the auxiliary control law (Step 4), which provides the optimal input sequence for a given artificial steady-state. The only requirement on the choice of $\epsilon_{s}$ is that $\mathcal{E}_{s} \triangleq\left\{x \mid\left\|x-\bar{x}_{s}\right\|_{P} \leq \epsilon_{s}\right\} \subseteq \bar{\chi}_{f}^{\operatorname{tr}}\left(\bar{x}_{s}, \bar{u}_{s}\right)$ for all $\left(\bar{x}_{s}, \bar{u}_{s}\right) \in \bar{\Theta}$, in order to ensure feasibility of the auxiliary control law. This choice of the warm-start input sequence allows for proving the existence of a feasible warm-start in Theorem 5.7.

Computation of $\alpha$ (Step 6): this step is crucial for providing a warm-start that satisfies the Lyapunov constraint. The artificial steady-state is moved as far as possible towards the real reference by means of the following minimization over $\alpha$ :

$$
\begin{array}{ll}
\alpha_{\min }= & \min _{\alpha \in[0,1]} \alpha \\
\text { s.t. } & \bar{x}_{N}^{\mathrm{ws}} \in \bar{x}_{f}^{\operatorname{tr}}\left(\bar{x}_{s}, \bar{u}_{s}\right), \\
& V_{N}^{\text {tr }}\left(\overline{\mathbf{x}}^{\mathrm{ws}}, \overline{\mathbf{u}}^{\mathrm{ws}}, \bar{x}_{s}, \bar{u}_{s}\right)+V_{f}\left(x_{\mathrm{nom}}^{\text {tr }}-\bar{x}_{0}^{\mathrm{ws}}\right) \leq \Pi_{\mathrm{prev}}^{\text {tr }} \\
& \text { for } \epsilon_{k}=\frac{1}{2}(1-\alpha)^{2}, \\
& \bar{x}_{s}=\alpha \bar{x}_{s}^{\tau, \operatorname{tr}}(x(k-1))+(1-\alpha) x_{r}, \\
& \bar{u}_{s}=\alpha \bar{u}_{s}^{\tau, \operatorname{tr}}(x(k-1))+(1-\alpha) u_{r} .
\end{array}
$$

If the optimal solution is $\alpha_{\min }<1$, the artificial steady-state $\left(\bar{x}_{s}^{\mathrm{ws}}\right.$, $\left.\bar{u}_{s}^{\text {ws }}\right)$ is improved by moving it from the previous one, i.e. $\left(\bar{x}_{s}^{\tau}(x(k-\right.$ $\left.1)), \bar{u}_{s}^{\tau}(x(k-1))\right)$, towards $\left(x_{r}, u_{r}\right)$ while guaranteeing satisfaction of the terminal constraint as well as the Lyapunov decrease constraint. Note that $\alpha=1$ is always a feasible solution to this optimization problem. While the warm-start tube center is not too close to the artificial steady-state, it can be shown that the choice of $\alpha_{\min }=1$, i.e. keeping the artificial steady-state at $\left(\bar{x}_{s}^{\tau}(x(k-1)), \bar{u}_{s}^{\tau}(x(k-1))\right)$ and applying the standard warm-start, still provides a sufficient decrease in the cost function to satisfy the Lyapunov constraint, and $\epsilon_{k}>0$ can therefore be chosen in Step 8. If the first tube center is close to $\bar{x}_{s}^{\tau}(x(k-1))$, the minimization in Step 6 will provide $\alpha_{\min }<1$, ensuring feasibility of the warm-start solution for $\epsilon_{k}=\frac{1}{2}(1-\alpha)^{2}$. The existence of a warm-start providing a strict cost decrease is therefore guaranteed in both cases and $\epsilon_{k}>0$ will be chosen at each time step in Step 8. This is proven in detail in Theorem 5.7, where also the particular choice of $\epsilon_{k}$ in the minimization is clarified.

Remark 5.4. The minimization of $\alpha$ in Step 6 of Algorithm 2 solving (12) can be rewritten in the following form by using $\rho=$ $1-\alpha$ :

$\max \left\{\rho \in[0,1] \mid \rho^{2}+a_{1} \rho+b_{1} \leq 0, \rho^{2}+a_{2} \rho+b_{2} \leq 0\right\}$.

The solution is obtained by computing the maximum separately for each constraint, where for each of them an analytical solution can be derived, and then taking the smaller of the two values.

Remark 5.5. The real-time procedure for tracking is obtained by replacing the warm-start in Step 1 of Algorithm 1 by Algorithm 2. In addition, Steps 6-8 of Algorithm 1 are modified as follows in order to ensure that the real-time cost can be upper bounded by a $\mathcal{K}_{\infty}$-class function of $\left\|x-x_{r}\right\|$ :

$$
\begin{aligned}
& \text { if }\left\|x-x_{r}\right\|_{P} \leq \epsilon_{f} \text { and } V_{N}^{t r}\left(\tilde{\mathbf{x}}, \tilde{\mathbf{u}}, \tilde{x}_{s}, \tilde{u}_{s}\right)+V_{f}\left(x(k)-\tilde{x}_{0}\right)>V_{f}\left(x(k)-x_{r}\right) \\
& \text { then } \\
& \text { un }=\left[u_{r}, \ldots, u_{r}\right], \tilde{x}_{0}=x_{r}, \tilde{x}_{s}=x_{r}, \tilde{u}_{s}=u_{r} \\
& \text { end if }
\end{aligned}
$$

where $\epsilon_{f}$ is now such that $\mathcal{E}_{f} \triangleq\left\{x \mid\left\|x-x_{r}\right\|_{P} \leq \epsilon_{f}\right\} \subseteq x_{r} \oplus \mathcal{Z}_{W}$.

Feasibility of the warm-start provided by Algorithm 2 for Problem $\mathbb{P}_{N}^{\tau, t r}(x)$ is proven in detail in the following in Theorem 5.7. This is key to showing convergence of the closed-loop system under the proposed $\tau$-RT control law for tracking to an RPI set around $x_{r}$ in Theorem 5.8. We first state a lemma that is required for this proof by showing that if the initial tube center $\bar{x}_{0}^{\text {ws }}$ is closer to the artificial steady-state $\bar{x}_{s}^{\tau, t r}(x(k-1))$ than some fraction of the distance between the artificial and the real steady-state $x_{r}$, then we can move the artificial steady-state towards $x_{r}$, while providing a decrease in the cost using the auxiliary control law. The proofs can be found in the appendix.

Lemma 5.6. Consider Problem $\mathbb{P}_{N}^{\tau, \text { tr }}(x)$. Let $\left(x_{r}, u_{r}\right)$ be a reference steady-state, $\left(\tilde{x}_{s}, \tilde{u}_{s}\right)$ a steady-state and $\tilde{x}_{0} \in \bar{X}_{f}^{\text {tr }}\left(\tilde{x}_{s}, \tilde{u}_{s}\right)$ a first tube center. Let $\tilde{\mathbf{u}}, \tilde{\mathbf{x}}$ be the input and state sequence generated by applying the auxiliary control law $\kappa_{f}^{\text {tr }}(x)=\tilde{u}_{s}+K\left(x-\tilde{x}_{s}\right)$ starting from $\tilde{x}_{0}$. Denote $\tilde{x}_{s, \delta}=\delta \tilde{x}_{s}+(1-\delta) x_{r}, \tilde{u}_{s, \delta}=\delta \tilde{u}_{s}+(1-\delta) u_{r}$. There exists a constant $\delta \in(0,1)$ such that if $\left\|\tilde{x}_{0}-\tilde{x}_{s}\right\|_{P} \leq(1-\delta)\left\|\tilde{x}_{s}-x_{r}\right\|_{P}$, then

$V_{N}^{\operatorname{tr}}\left(\tilde{\mathbf{x}}, \tilde{\mathbf{u}}, \tilde{x}_{s, \delta}, \tilde{u}_{s, \delta}\right) \leq V_{N}^{\operatorname{tr}}\left(\tilde{\mathbf{x}}, \tilde{\mathbf{u}}, \tilde{x}_{s}, \tilde{u}_{s}\right)-(1-\delta)^{2}\left\|\tilde{x}_{s}-x_{r}\right\|_{P}^{2}$.

Theorem 5.7 (Feasibility Warm-Start for Tracking). Let $\overline{\mathbf{u}}^{\tau, t r}(x(k-$ $1)), \bar{x}_{0}^{\tau, \operatorname{tr}}(x(k-1)), \bar{x}_{s}^{\tau, \operatorname{tr}}(x(k-1)), \bar{u}_{s}^{\tau, \operatorname{tr}}(x(k-1))$ be a feasible control sequence, tube center and artificial steady-state for Problem $\mathbb{P}_{N}^{\tau, t r}(x(k-1))$. The warm-start solution provided by Algorithm 2 is feasible for $\mathbb{P}_{N}^{\tau, \text { tr }}(x(k))$, where $x(k) \in A x(k-1)+B \kappa^{\tau, \operatorname{tr}}(x(k-$ 1)) $\oplus$ W, i.e. $\bar{x}_{0}^{w s} \in \bar{x}_{0}(x(k)),\left(\bar{x}_{s}^{w s}, \bar{u}_{s}^{w s}\right) \in \bar{\Theta}, \overline{\mathbf{u}}^{\text {ws }} \in \bar{u}_{N}^{\text {tr }}\left(\bar{x}_{0}^{w s}\right.$, $\left.\bar{x}_{s}^{w s}, \bar{u}_{s}^{w s}\right)$.

We can now show that by using the modified warm-start in Algorithm 2 the results for regulation presented in Section 4 extend to the tracking case and feasibility again implies stability. 
Theorem 5.8 (Convergence Under $\kappa^{\tau, t r}(x)$ ). Consider Problem $\mathbb{P}_{N}^{\tau, t r}(x)$ fulfilling Assumption 5.3, where $\left(x_{r}, u_{r}\right) \in \bar{\Theta}$ is a feasible steady-state. The closed-loop system (1) under the $\tau$-RT control law in (10) that is obtained from Algorithms 1 and 2 converges to an RPI set around $x_{r} \forall x \in X_{N}^{\text {tr }}$, i.e. the system $x(k+1)-x_{r}=A\left(x(k)-x_{r}\right)+$ $B\left(\kappa^{\tau, \operatorname{tr}}(x(k))-u_{r}\right)+w(k)$ is ISS in $X_{N}^{\text {tr }}$ with respect to $w(k) \in \mathcal{W}$.

Proof. The proof follows similar arguments as the proof of Theorem 4.7 for showing that $V^{\tau, \operatorname{tr}}(x)$ is an ISS Lyapunov function. By convexity of $\|\cdot\|_{Q}^{2}$, there exists a $\mathcal{K}_{\infty}$-class function $\underline{\alpha}(\cdot)$ such that $V^{\tau, \operatorname{tr}}(x) \geq \frac{1}{2}\left\|x-\bar{x}_{s}^{\tau}(x)\right\|_{Q}^{2}+\frac{1}{2} c_{T}\left\|\bar{x}_{s}^{\tau}(x)-x_{r}\right\|_{Q}^{2} \geq \frac{1}{4} c_{T}\left\|x-x_{r}\right\|_{Q}^{2} \geq$ $\underline{\alpha}\left(\left\|x-x_{r}\right\|\right) \forall x \in X_{N}^{\text {tr }}$, where $c_{T} \leq 1$ is such that $T_{x} \succeq c_{T} Q$. Steps 6-8 (see Remark 5.5) ensure the upper bound and the existence of a $\mathcal{K}_{\infty}$-class function $\bar{\alpha}(\cdot)$ such that $V^{\tau, t r}(x) \leq V_{f}\left(x-x_{r}\right) \leq$ $\bar{\alpha}\left(\left\|x-x_{r}\right\|\right) \forall x \in \mathcal{E}_{f}$. Following the same argument as in the proof of Theorem 4.7 and replacing $\epsilon$ with $\epsilon_{\min }=\min _{k \in \mathbb{N}} \epsilon_{k}>0$, it can be shown that there exists a $\mathcal{K}_{\infty}$-class function $\beta(\cdot)$ and a $\mathcal{K}$-class function $\gamma(\cdot)$ such that $V_{L}^{\tau, \operatorname{tr}}(x(k))-V^{\tau, \operatorname{tr}}(x(k-1)) \leq$ $-\beta\left(\left\|x(k-1)-x_{r}\right\|\right)+\gamma(\|w\|)$, concluding the proof.

Remark 5.9. Note that Theorems 5.7 and 5.8 prove the existence of a (ISS) Lyapunov function for the tracking approach proposed in Limon et al. (2008) also for the optimal case, which, according to the authors' knowledge, has not been presented in the literature before. The availability of a Lyapunov function can be crucial for providing stability guarantees for extensions of the tracking approach, such as the presented real-time case.

Remark 5.10. At the first optimization step after a reference change, Problem $\mathbb{P}_{N}^{\tau, \text { tr }}(x)$ without the Lyapunov constraint (9f) has to be considered in the real-time procedure since the reference change may increase the cost value compared to the previous solution and a cost decrease cannot be enforced.

Remark 5.11. The use of an artificial reference and corresponding target set enlarges the domain of attraction compared to a standard MPC approach for reference tracking and $x_{N}^{\text {tr }} \supseteq \chi_{N}$ (Limon et al., 2008).

Remark 5.12. $V_{o}(\cdot, \cdot)$ is chosen as a quadratic function and does not represent an exact penalty function (Luenberger, 1984) since this work focuses on a suboptimal method. Local optimality is hence not guaranteed, i.e. the optimal artificial reference resulting from $\mathbb{P}_{N}^{\tau, \text { tr }}(x)$ might differ from the desired reference, although $x_{s}^{*}=x_{r}, u_{s}^{*}=u_{r}$ is a feasible solution and could be enforced. This optimality loss can be reduced by choosing large weight matrices $T_{x}$ and $T_{u}$. However, all results on real-time MPC for reference tracking presented in this paper directly extend to the use of $1-/ \infty$-norms in the offset cost, representing an exact penalty function for sufficiently large weights $T_{x}$ and $T_{u}$ (Ferramosca, Limon, Alvarado, Alamo, \& Camacho, 2009; Luenberger, 1984). Note that the choice of $V_{o}(\cdot, \cdot)$ only affects the transient behavior and not the optimal steady-state.

Having set the theoretical background, the remaining sections outline some implementation details of the proposed real-time MPC approach and show that computation times in the range of milliseconds can be achieved even for the more complex tracking case.

\section{Implementation}

In this section we discuss implementation aspects of the realtime robust MPC problem for tracking $\mathbb{P}_{N}^{\tau, t r}(x)$. For a given value $x \in X_{N}^{\text {tr }}$ problem $\mathbb{P}_{N}^{\tau, t r}(x)$ can be written as a convex QCQP with affine equality constraints and affine and quadratic inequality constraints, where the optimization variables are given by all states and inputs over the horizon as well as the artificial steady-state.

A feasible start interior-point method is applied in this work, which provides feasibility at all times and can efficiently solve QCQPs of the given form (Nocedal \& Wright, 2006). The main computational effort in an interior-point method is the Newton step computation, see e.g. Nocedal and Wright (2006) for more details. However, the results on structure exploitation in MPC methods presented in the literature, e.g. Wang and Boyd (2010), Wright (1997), cannot be directly applied in the considered case, due to the fact that the Lyapunov constraint and the cost of the tracking formulation introduce coupling across the horizon and thereby significantly modify the structure of the resulting Newton system. There are two main options to exploit the new structure in the real-time problem:

A: by reordering, the matrix in the Newton step can be brought into an arrow structure with a dense band of size $2 n+m+$ 1. Variable elimination reducing the system to the so-called augmented form, see e.g. Domahidi et al. (2012), Nocedal and Wright (2006), can then be applied, where the key is not to eliminate the Lagrange multiplier associated with the Lyapunov constraint, which would result in a dense Newton system. More details on this re-ordering and how to exploit this structure can be found in Zeilinger (2011).

$\mathrm{B}$ : by adding additional variables, the bandedness of the matrix in the Newton step can be recovered:

$$
\begin{aligned}
& \left\|x_{\text {nom }}^{\text {tr }}-\bar{x}_{0}\right\|_{P}^{2}+\left\|\bar{x}_{0}-\bar{x}_{s, 0}\right\|_{Q}^{2}+\left\|\bar{x}_{s, 0}-x_{r}\right\|_{T_{x}}^{2} \leq \gamma_{0}, \\
& \left\|\bar{u}_{0}-\bar{u}_{s, 0}\right\|_{R}^{2}+\left\|\bar{u}_{s, 0}-u_{r}\right\|_{T_{u}}^{2} \leq \delta_{0}, \\
& \left\|\bar{x}_{i}-\bar{x}_{s, i}\right\|_{Q}^{2} \leq \gamma_{i}, \quad\left\|\bar{u}_{i}-\bar{u}_{s, i}\right\|_{R}^{2} \leq \delta_{i}, \quad i=1, \ldots, N-1 \\
& \left\|\bar{x}_{N}-\bar{x}_{s, N}\right\|_{P}^{2}-J_{N} \leq 0, \\
& J_{0}=\Pi_{\mathrm{prev}}^{t r}, \quad J_{i+1}=J_{i}-\gamma_{i}-\delta_{i}, \quad i=1, \ldots, N-1, \\
& \bar{x}_{s, i+1}=\bar{x}_{s, i}, \quad \bar{u}_{s, i+1}=\bar{u}_{s, i}, \quad i=0, \ldots, N-1 .
\end{aligned}
$$

The Newton step can then be computed using the existing results, e.g. Rao, Wright, and Rawling (1998), Wang and Boyd (2010), or the recent results in Domahidi et al. (2012) that are tailored to MPC with quadratic constraints. The number of optimization variables can be reduced by employing a parametrization of the steady-state $x_{s}=M_{x} \theta, u_{s}=M_{u} \theta$, see Limon et al. (2008) for more details.

\section{Numerical examples}

The presented techniques are demonstrated in the following by numerical examples. The real-time problems have been implemented using the FORCES code generator (Domahidi, 2012; Domahidi et al., 2012). A primal-dual interior-point method is applied implementing Mehrotra's predictor-corrector method, which is adapted to ensure primal feasibility (Nocedal \& Wright, 2006). Concerning the warm-start of the slack and dual variables, we take the slacks of the primal warm-start solution and the dual variables such that an initially chosen complementarity measure is satisfied. Note that any other feasible warm-start method, e.g. the improved dual warm-start proposed in Shahzad and Goulart (2011), could also be applied. The offline set computations were carried out using the YALMIP toolbox (Löfberg, 2004) and the solver SDPT3 (Toh, Todd, \& Tütüncü, 1999).

A system of oscillating masses (Wang \& Boyd, 2010) is chosen to examine the proposed real-time methods for regulation and tracking. The masses are interconnected by springs and dampers and are connected to walls on the side. The actuators exert tension between two neighboring masses. A system of three masses is illustrated in Fig. 2, which can similarly be extended to an increasing number of masses using the same structure. 


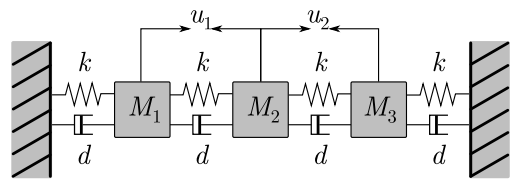

Fig. 2. Three masses example.

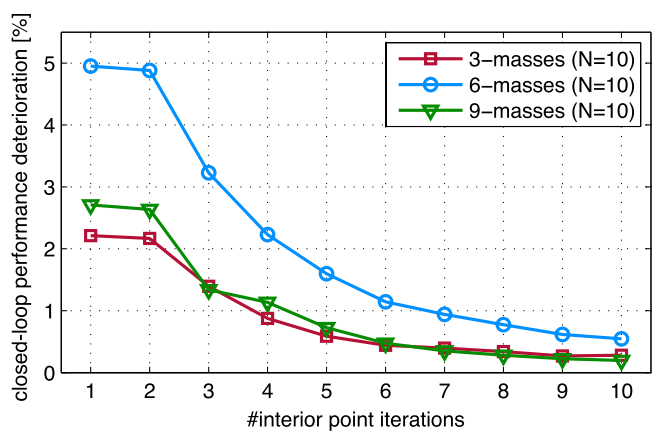

Fig. 3. Average closed-loop performance deterioration for the real-time MPC approach over 100 randomly sampled initial values.

\subsection{Regulation}

We first consider the real-time regulation problem described in Section 4. The masses have value $1 \mathrm{~kg}$, the spring constant is $k=0.9 \mathrm{~N} / \mathrm{m}$ and the damping constant $d=0.1 \mathrm{~N} \mathrm{~s} / \mathrm{m}$. The control inputs are constrained to lie in $\pm 1 \mathrm{~N}$ and the displacement of the masses is constrained in $\pm 4 \mathrm{~m}$. The system is discretized with sampling time $t_{\mathrm{s}}=0.5 \mathrm{~s}$. An additive random disturbance with $\|w(k)\|_{2} \leq 0.03$ is acting on the system.

The performance of the real-time procedure and the effect of the number of performed interior-point iterations per solve are investigated using systems of three, six and nine masses. The average closed-loop performance deterioration when applying the suboptimal controller $\hat{\kappa}\left(x_{i}\right)$ with respect to the closed-loop cost using the optimal MPC control law $\kappa(x)$ is given by $\Delta J_{\mathrm{cl}}=$ $\frac{\sum_{i=0}^{\infty}\left(l\left(x_{i}, \hat{\kappa}\left(x_{i}\right)\right)-l\left(x_{i}, \kappa\left(x_{i}\right)\right)\right)}{\sum_{i=0}^{\infty} l\left(x_{i}, \kappa\left(x_{i}\right)\right)}$, which is estimated by simulating the trajectory for a long time period and taking the mean over 100 randomly sampled initial states. The results in Fig. 3 show that a three step solution still shows considerably low performance loss of about 3\% or less in all three example problems. Putting these results into perspective, using a cold-start approach (all primal variables initialized to zero and the dual variables to 1) for the same initial states, there exist initial states that lead to infeasibilities in the closed-loop simulation for up to 10 interior-point iterations and for up to 4 iterations more than $50 \%$ of all initial states result in infeasibilities.

The computation time for Algorithms 1 and 2 is mainly given by the optimization, i.e. the time to perform the interior-point iterations. A detailed computational comparison of the implemented real-time method for regulation against other solvers applied to MPC problems without any guarantees for a range of problem sizes can be found in Domahidi et al. (2012), showing that fast sampling rates can be achieved for the real-time formulation. For example, for the six masses system with a horizon $N=10$, three iterations can be computed in less than $0.4 \mathrm{~ms}$ (Desktop PC, Intel i7, 3.2 GHz, 12 GB RAM, running Ubuntu 10.04 using a single core). This demonstrates the advantage of the proposed real-time MPC approach, where for instance even three iterations provide not only high performance but also guaranteed feasibility and stability.

\subsection{Tracking}

In the following, we use the same example problems of three, six and nine masses to demonstrate the real-time procedure for
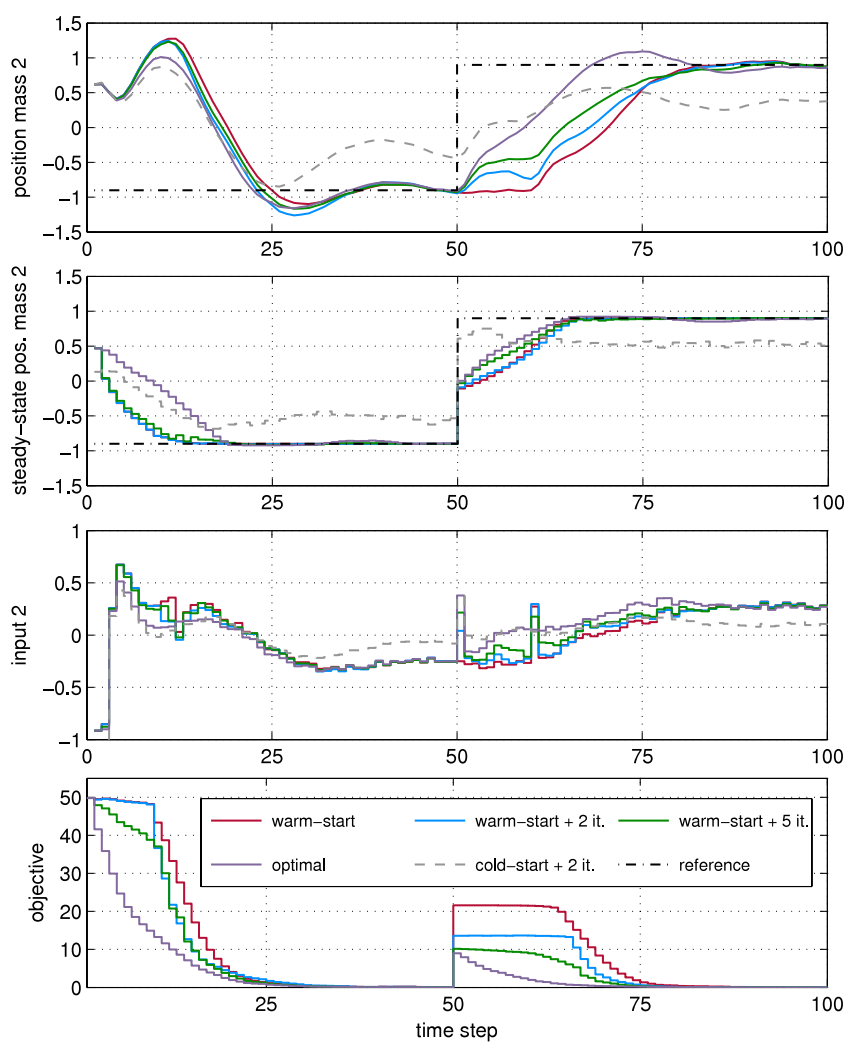

Fig. 4. Comparison of the closed-loop performance using the warm-start solution, the suboptimal solution after 2 and 5 online iterations (with warm-start), the suboptimal solution after 2 iterations using cold-start from the origin, and the optimal solution.

tracking introduced in Section 5. First, consider the system of three masses from Fig. 2, with $k=0.3 \mathrm{Nm}$ and $d=0.1 \mathrm{~N} \mathrm{~s} / \mathrm{m}$. The inputs are again constrained to lie in $\pm 1 \mathrm{~N}$ and the displacement of the masses in $\pm 4 \mathrm{~m}$. The horizon length is chosen as $N=10$ and a worst-case disturbance with $\|w(k)\|_{2}=0.02$ is acting on the system. At the beginning of the simulation, the tracking reference is a displacement of the second mass of $-0.9 \mathrm{~m}$ (and no displacement of the first and third mass). At time step $k=50$, a step change to $+0.9 \mathrm{~m}$ in the reference position of the second mass is applied. Fig. 4 shows the simulated closed-loop trajectories of the position of the second mass, the corresponding steadystate, the second input and the value of the Lyapunov function for different starting strategies and number of optimization steps. We compare the closed-loop performance of applying the warmstart solution without any optimization and with two and five interior-point steps to a cold-start solution with two iterations and the optimal solution. The simulation illustrates the role of the artificial reference. While directly using the new reference of $+0.9 \mathrm{~m}$ starting from position $-0.9 \mathrm{~m}$ would be infeasible, the artificial reference is chosen to ensure recursive feasibility and then converges to the desired reference.

The results demonstrate that only applying the warm-start solution already provides convergence to the desired reference, even after the step change. This is enabled by the proposed warm-start procedure in Algorithm 2, which includes a warmstart of the artificial steady-state, moving it towards the desired target. This is particularly evident after the step change, where the minimization of $\alpha$ moves the artificial reference to $-0.1 \mathrm{~m}$. The Lyapunov function decreases monotonically proving asymptotic convergence of all masses to the desired position. Note that the increase in the Lyapunov function at $k=50$ is caused by the reference change, which is enabled by resetting the Lyapunov constraint. Since the warm-start is given by the shifted input 
Table 1

Computation times for one interior-point iteration solving the tracking problem $\mathbb{P}_{N}^{\tau, t r}$ for a horizon length of $N=10$ on a MacBook Pro with Intel Core i7 CPU at $2.6 \mathrm{GHz}$.

\begin{tabular}{lrlccc}
\hline \#masses & $n$ & $m$ & $\operatorname{Min}(\mu \mathrm{s})$ & Mean $(\mu \mathrm{s})$ & $\operatorname{Max}(\mu \mathrm{s})$ \\
\hline 3 & 6 & 2 & 53.95 & 55.90 & 58.52 \\
6 & 12 & 5 & 189.75 & 195.77 & 231.83 \\
9 & 18 & 8 & 479.63 & 498.74 & 544.68 \\
\hline
\end{tabular}

sequence, a delay of $N-1$ steps occurs after the artificial reference is moved at $k=50$, until the control inputs react to the reference change. This behavior could be improved by small adaptations in the warm-start procedure, but the effect is automatically mitigated by performing a few optimization steps. This can be seen in Fig. 4 , where the control input for only two optimization steps already reacts at $k=50$.

Comparing the performance of the warm-start without optimization with two and five online iterations, we can see that the performance gradually improves with the number of performed iterations. The closed-loop performance deterioration with respect to the optimal closed-loop trajectory applying the warm-start without optimization is $16.60 \%$, for two iterations it is $15.58 \%$ and for five iterations $5.61 \%$. This shows that only five online optimization steps already provide very good performance. The cold-start method with two online iterations, in contrast, results in an input constraint violation at the time step $k=1$ and is not able to track the desired reference. Note that the cost function cannot be compared in this case, since the resulting solutions are infeasible for the constraints of the optimization problem.

We investigate the computation times for the tracking method by recording the time to perform one iteration of the online optimization. The minimum, average and maximum times are given in Table 1, demonstrating that the real-time tracking method can be implemented for sampling times below milliseconds. For example, for the three masses system, five iterations can be computed in less than $300 \mu \mathrm{s}$.

\section{Conclusions}

A new approach for real-time robust MPC was presented that provides guarantees on feasibility and input-to-state stability for arbitrary real-time constraints. The proposed technique is based on robust MPC design, a stability enforcing constraint and a warmstart procedure. As a key result, a new warm-start method and (ISS) Lyapunov function for real-time tracking are introduced. Since stability is guaranteed by design, the performance can be traded off against the computation time. The method enables realtime regulation and tracking for linear systems of any practical dimension, and the presented numerical examples demonstrate that computation times in the range of milliseconds can be achieved.

\section{Acknowledgments}

The authors would like to thank Stephen Boyd and Yang Wang for their valuable discussions.

\section{Appendix A. Proof of Lemma 4.6}

Let $\Delta x_{0}^{-}=x^{-}-\bar{x}_{0}^{\tau}\left(x^{-}\right)$. Feasibility of the warm-start for Problem $\mathbb{P}_{N}^{\tau}(x)$ without the Lyapunov constraint (5f) was shown in Mayne et al. (2005). In order to prove satisfaction of (5f) we make use of the following fact: $x_{\text {nom }}=\bar{x}_{1}^{\tau}\left(x^{-}\right)+(A+B K)\left(x^{-}-\bar{x}_{0}^{\tau}\left(x^{-}\right)\right)$. From Assumption 4.3, $\bar{x}_{0}^{\text {ws }}=\bar{x}_{1}^{\tau}\left(x^{-}\right)$and standard arguments in
MPC, we get

$$
\begin{aligned}
& V_{N}\left(\overline{\mathbf{x}}^{\mathrm{ws}}, \overline{\mathbf{u}}^{\mathrm{ws}}\right)-V_{N}\left(\overline{\mathbf{x}}^{\tau}\left(x^{-}\right), \overline{\mathbf{u}}^{\tau}\left(x^{-}\right)\right)+V_{f}\left(x_{\mathrm{nom}}-\bar{x}_{0}^{\mathrm{ws}}\right)-V_{f}\left(\Delta x_{0}^{-}\right) \\
& \quad \leq-\left\|\bar{x}_{0}^{\tau}\left(x^{-}\right)\right\|_{Q}^{2}+V_{f}\left((A+B K)\left(\Delta x_{0}^{-}\right)\right)-V_{f}\left(\Delta x_{0}^{-}\right) \\
& \quad \leq-\left\|\bar{x}_{0}^{\tau}\left(x^{-}\right)\right\|_{Q}^{2}-\left\|\Delta x_{0}^{-}\right\|_{Q}^{2} \leq-\frac{1}{2}\left\|x^{-}\right\|_{Q}^{2}
\end{aligned}
$$

where the last step follows from Assumptions 4.3 and 4.4 and convexity of $\|\cdot\|_{Q}^{2}$ providing $\frac{1}{2}\|x+y\|_{Q}^{2} \leq\|x\|_{Q}^{2}+\|y\|_{Q}^{2}$, proving the result.

\section{Appendix B. Proof of Lemma 5.6}

We denote $A_{K} \triangleq A+B K, \Delta \tilde{x}_{0} \triangleq \tilde{x}_{0}-\tilde{x}_{s}, \Delta \tilde{x}_{s} \triangleq \tilde{x}_{s}-\tilde{x}_{r}$, $\Delta \tilde{u}_{s} \triangleq \tilde{u}_{s}-\tilde{u}_{r}, \rho=1-\delta$. By Assumption 5.3, $V_{f}(x)$ is a Lyapunov function and $P \succeq Q+K^{T} R K$, therefore $\left\|A_{K}^{i} x\right\|_{Q} \leq\left\|A_{K}^{i} x\right\|_{P} \leq\|x\|_{P}$ and $\left\|A_{K}^{i} x\right\|_{K^{T} R K} \leq\left\|A_{K}^{i} x\right\|_{P} \leq\|x\|_{P}$. From the use of the auxiliary control law, i.e. $\tilde{u}_{i}=K A_{K}^{i} \Delta \tilde{x}_{0}+\tilde{u}_{s},\left\|\Delta \tilde{x}_{0}\right\|_{P} \leq \rho\left\|\Delta \tilde{x}_{s}\right\|_{P}$ and recalling that $\tilde{x}_{s}=A \tilde{x}_{s}+B \tilde{u}_{s}$ we obtain

$$
\begin{aligned}
l\left(\tilde{x}_{i}-\right. & \left.\tilde{x}_{s, \delta}, \tilde{u}_{i}-\tilde{u}_{s, \delta}\right)-l\left(\tilde{x}_{i}-\tilde{x}_{s}, \tilde{u}_{i}-\tilde{u}_{s}\right) \\
= & \left\|A_{K}^{i} \Delta \tilde{x}_{0}+\rho \Delta \tilde{x}_{s}\right\|_{Q}^{2}+\left\|K A_{K}^{i} \Delta \tilde{x}_{0}+\rho \Delta \tilde{u}_{s}\right\|_{R}^{2} \\
& \quad-\left\|A_{K}^{i} \Delta \tilde{x}_{0}\right\|_{Q}^{2}-\left\|K A_{K}^{i} \Delta \tilde{x}_{0}\right\|_{R}^{2} \\
\leq & 2 \rho\left\|A_{K}^{i} \Delta \tilde{x}_{0}\right\|_{Q}\left\|\Delta \tilde{x}_{s}\right\|_{Q}+\rho^{2}\left\|\Delta \tilde{x}_{s}\right\|_{Q}^{2} \\
& +2 \rho\left\|A_{K}^{i} \Delta \tilde{x}_{0}\right\|_{K^{T} R K}\left\|\Delta \tilde{u}_{s}\right\|_{R}+\rho^{2}\left\|\Delta \tilde{u}_{s}\right\|_{R}^{2} \\
\leq & 3 \rho^{2}\left\|\Delta \tilde{x}_{s}\right\|_{P}^{2}+2 \rho^{2}\left\|\Delta \tilde{x}_{s}\right\|_{P}\left\|\Delta \tilde{u}_{s}\right\|_{R}+\rho^{2}\left\|\Delta \tilde{u}_{s}\right\|_{R}^{2}
\end{aligned}
$$

and similarly $V_{f}\left(\tilde{x}_{N}-\tilde{x}_{s, \delta}\right)-V_{f}\left(\tilde{x}_{N}-\tilde{x}_{s}\right) \leq 3 \rho^{2}\left\|\Delta \tilde{x}_{s}\right\|_{P}^{2}$. From convexity of $V_{o}(\cdot, \cdot)$ we obtain $V_{o}\left(\tilde{x}_{s, \delta}-x_{r}, \tilde{u}_{s, \delta}-u_{r}\right) \leq$ $\delta V_{o}\left(\Delta \tilde{x}_{s}, \Delta \tilde{u}_{s}\right)$ and therefore

$$
\begin{aligned}
& V_{N}^{\text {tr }}\left(\tilde{\mathbf{x}}, \tilde{\mathbf{u}}, \tilde{x}_{s, \delta}, \tilde{u}_{s, \delta}\right)-V_{N}^{\text {tr }}\left(\tilde{\mathbf{x}}, \tilde{\mathbf{u}}, \tilde{x}_{s}, \tilde{u}_{s}\right)+\rho^{2}\left\|\Delta \tilde{x}_{s}\right\|_{P}^{2} \\
& \leq \rho\left[3(N+1) \rho\left\|\Delta \tilde{x}_{s}\right\|_{P}^{2}+2 N \rho\left\|\Delta \tilde{x}_{s}\right\|_{P}\left\|\Delta \tilde{u}_{s}\right\|_{R}\right. \\
& \left.\quad+N \rho\left\|\Delta \tilde{u}_{s}\right\|_{R}^{2}+\rho\left\|\Delta \tilde{x}_{s}\right\|_{P}^{2}-V_{o}\left(\Delta \tilde{x}_{s}, \Delta \tilde{u}_{s}\right)\right] \leq 0,
\end{aligned}
$$

which is satisfied for

$$
0<\rho \leq \frac{V_{o}\left(\Delta \tilde{x}_{S}, \Delta \tilde{u}_{S}\right)}{(3 N+4)\left\|\Delta \tilde{x}_{s}\right\|_{P}^{2}+2 N\left\|\Delta \tilde{x}_{s}\right\|_{P}\left\|\Delta \tilde{u}_{s}\right\|_{R}+N\left\|\Delta \tilde{u}_{s}\right\|_{R}^{2}} \text { and hence } \delta<1,
$$
proving the result.

\section{Appendix C. Proof of Theorem 5.7}

For ease of notation we omit the dependence of the real-time solution on $x^{-}$and denote $\Delta \bar{x}_{0}^{\tau, t r} \triangleq \bar{x}_{0}^{\tau, t r}-\bar{x}_{s}^{\tau, t r}, \Delta \bar{x}_{s}^{\tau, t r} \triangleq \bar{x}_{s}^{\tau, t r}-x_{r}$, $\Delta \bar{u}_{0}^{\tau, t r} \triangleq \bar{u}_{0}^{\tau, t r}-\bar{u}_{s}^{\tau, t r}$ and $A_{K} \triangleq A+B K$. Feasibility for $\mathbb{P}_{N}^{\tau, t r}(x(k))$ without the Lyapunov constraint (9f) follows from feasibility of the shifted sequence (Limon et al., 2008, 2010) or feasibility of the auxiliary control law. Since the minimization of $\alpha$ in Step 6 enforces the terminal constraint, satisfaction of the state and input constraints is maintained and since $\bar{\Theta}$ is a convex set, feasibility of $\left(x_{r}, u_{r}\right)$ and $\left(\bar{x}_{s}^{\tau, t r}, \bar{u}_{s}^{\tau, t r}\right)$ imply $\left(\bar{x}_{s}^{\text {ws }}, \bar{u}_{s}^{\text {ws }}\right) \in \bar{\Theta}$ for $\alpha \in[0,1]$. The important condition to prove is hence feasibility of the Lyapunov constraint (9f). The following facts are used: $x_{\text {nom }}^{\text {tr }}=\bar{x}_{0}^{\text {ws }}+A_{K}\left(x^{-}-\right.$ $\left.\bar{x}_{0}^{\tau, t r}\right), \frac{1}{2}\|x+y\|_{Q}^{2} \leq\|x\|_{Q}^{2}+\|y\|_{Q}^{2}$, which is provided by convexity of $\|\cdot\|_{Q}^{2}$, and by Assumption 5.3: $V_{f}\left(x_{\text {nom }}^{\text {tr }}-\bar{x}_{0}^{\text {ws }}\right)-V_{f}\left(x^{-}-\bar{x}_{0}^{\tau, t r}\right)=$ $\left\|A_{K}\left(x^{-}-\bar{x}_{0}^{\tau, \text { tr }}\right)\right\|_{P}^{2}-\left\|x^{-}-\bar{x}_{0}^{\tau, t r}\right\|_{P}^{2} \leq-\left\|x^{-}-\bar{x}_{0}^{\tau, \text { tr }}\right\|_{Q}^{2}$. If $\alpha_{\min }<1$ in Step 6, the Lyapunov constraint is satisfied by construction with $\epsilon_{k}=\frac{1}{2}\left(1-\alpha_{\min }\right)^{2}>0$. It therefore has to be shown that either $\alpha_{\text {min }}<1$, or there exists an $\epsilon_{k}>0$ such that (9f) is satisfied even if $\alpha_{\min }=1$ is the optimal solution in Step 6 . We prove the result considering the following two cases:

Case 1: $\left\|\bar{x}_{0}^{\mathrm{ws}}-\bar{x}_{s}^{\tau, \text { tr }}\right\|_{P} \geq \epsilon_{s}$.

In this case it will be shown that $\epsilon_{k}>0$ is satisfied by the warm-start even if $\alpha_{\min }=1$. $\overline{\mathbf{u}}^{\text {ws }}$ is given by the shifted sequence 
in Step 2 of Algorithm 2, $\overline{\mathbf{x}}^{\mathrm{ws}}$ is the corresponding state sequence starting from $\bar{x}_{0}^{\mathrm{ws}}=\bar{x}_{1}^{\tau, \text { tr }}$ and $\bar{x}_{s}^{\mathrm{ws}}=\bar{x}_{s}^{\tau, t r}, \bar{u}_{s}^{\mathrm{ws}}=\bar{u}_{s}^{\tau, t r}$. Let $\Delta V=V_{N}^{t r}\left(\overline{\mathbf{x}}^{\mathrm{ws}}, \overline{\mathbf{u}}^{\mathrm{ws}}, \bar{x}_{s}^{\mathrm{ws}}, \bar{u}_{s}^{\mathrm{ws}}\right)+V_{f}\left(x_{\mathrm{nom}}^{t r}-\bar{x}_{0}^{\mathrm{ws}}\right)-V_{N}^{t r}\left(\overline{\mathbf{x}}^{\tau, t r}, \overline{\mathbf{u}}^{\tau, t r}\right.$, $\left.\bar{x}_{s}^{\tau, t r}, \bar{u}_{s}^{\tau, t r}\right)-V_{f}\left(x^{-}-\bar{x}_{0}^{\tau, t r}\right)$. This warm-start yields the cost decrease

$$
\begin{aligned}
\Delta V & \leq-\left\|\Delta \bar{x}_{0}^{\tau, t r}\right\|_{Q}^{2}-\left\|\Delta \bar{u}_{0}^{\tau, t r}\right\|_{R}^{2}-\left\|x^{-}-\bar{x}_{0}^{\tau, \text { tr }}\right\|_{Q}^{2} \\
& \leq-\epsilon_{k}\left\|\bar{x}_{0}^{\tau, t r}-x_{r}\right\|_{Q}^{2}-\left\|x^{-}-\bar{x}_{0}^{\tau, t r}\right\|_{Q}^{2} \\
& \leq-\frac{1}{2} \epsilon_{k}\left\|x^{-}-x_{r}\right\|_{Q}^{2},
\end{aligned}
$$

where $\epsilon_{k} \triangleq \min \left(\left(\left\|\Delta \bar{x}_{0}^{\tau, \text { tr }}\right\|_{Q}^{2}+\left\|\Delta \bar{u}_{0}^{\tau, \text { tr }}\right\|_{R}^{2}\right) /\left\|\bar{x}_{0}^{\tau, \text { tr }}-x_{r}\right\|_{Q}^{2}, 1\right)$. By the definition of $\bar{x}_{0}^{\mathrm{ws}},\|x\|_{P}=\left\|P^{\frac{1}{2}} x\right\|_{2}$ and by using the steadystate condition we obtain: $\epsilon_{S} \leq\left\|A\left(\Delta \bar{x}_{0}^{\tau, t r}\right)+B\left(\Delta \bar{u}_{0}^{\tau, t r}\right)\right\|_{P} \leq$ $\|A\|_{P}\left\|\Delta \bar{x}_{0}^{\tau, t r}\right\|_{2}+\|B\|_{P}\left\|\Delta \bar{u}_{0}^{\tau, t r}\right\|_{2}$. Therefore either

$$
\begin{aligned}
& \|B\|_{P}\left\|\Delta \bar{u}_{0}^{\tau, \text { tr }}\right\|_{2} \leq 0.5 \epsilon_{s} \\
& \quad \Rightarrow\|A\|_{P}\left\|\Delta \bar{x}_{0}^{\tau, t r}\right\|_{2} \geq 0.5 \epsilon_{s} \Rightarrow\left\|\Delta \bar{x}_{0}^{\tau, t r}\right\|_{Q}^{2} \geq \bar{\epsilon}_{s}^{2} \\
& \quad \text { or }\|B\|_{P}\left\|\Delta \bar{u}_{0}^{\tau, t r}\right\|_{2} \geq 0.5 \epsilon_{s} \Rightarrow\left\|\Delta \bar{u}_{0}^{\tau, t r}\right\|_{R}^{2} \geq \bar{\epsilon}_{s}^{2},
\end{aligned}
$$

where $\bar{\epsilon}_{s}=\frac{0.5 \epsilon_{s}}{\sqrt{c} \max \left(\|A\|_{P},\|B\|_{P}\right)}$ and $c \geq 1$ is such that $c Q \succeq I$, $c R \succeq I$. As a result $\bar{\epsilon}_{s}^{2} \leq\left\|\Delta \bar{x}_{0}^{\tau, \text { tr }}\right\|_{Q}^{2}+\left\|\Delta \bar{u}_{0}^{\tau, \text { tr }}\right\|_{R}^{2}$ and the Lyapunov constraint is satisfied with $\epsilon_{k} \geq \min \left(\bar{\epsilon}_{s}^{2} /\left\|\bar{x}_{0}^{\tau, t r}-x_{r}\right\|_{Q}^{2}, 1\right) \geq$ $\min \left(\bar{\epsilon}_{s}^{2} / \max _{x \in \mathbb{X}}\left\|x-x_{r}\right\|_{Q}^{2}, 1\right)>0$.

Case 2: $\left\|\bar{x}_{0}^{\mathrm{ws}}-\bar{x}_{s}^{\tau, t r}\right\|_{P}<\epsilon_{s}$.

In this case $\bar{x}_{0}^{\text {ws }} \in \bar{X}_{f}^{\operatorname{tr}}\left(\bar{x}_{s}^{\tau, t r}, \bar{u}_{s}^{\tau, t r}\right)$, i.e. the initial tube center is close to the artificial steady-state, and the optimal sequence to regulate the tube centers to the steady-state $\left(\bar{x}_{s}^{\tau, t r}, \bar{u}_{s}^{\tau, t r}\right)$ is by applying the auxiliary control law $\kappa_{f}^{\text {tr }}(x)=\bar{u}_{s}^{\tau, t r}+K\left(x-\bar{x}_{s}^{\tau, t r}\right)$. By the optimality of the auxiliary control law providing a lower cost than the shifted sequence, (C.1a) again holds. We will show in the following that if there exists a constant $\delta \in(0,1)$ according to Lemma 5.6, such that $\left\|\bar{x}_{0}^{\text {ws }}-\bar{x}_{s}^{\tau, \text { tr }}\right\|_{P} \geq(1-\delta)\left\|\Delta \bar{x}_{s}^{\tau, \text { tr }}\right\|_{P}$, then $\epsilon_{k}>0$ even if $\alpha_{\min }=1$, or if $\left\|\bar{x}_{0}^{\text {ws }}-\bar{x}_{s}^{\tau, \text { tr }}\right\|_{P} \leq(1-\delta)\left\|\Delta \bar{x}_{s}^{\tau, t r}\right\|_{P}$, then the optimization of $\alpha$ in Step 6 always provides $\alpha_{\min }<1$.

Case 2a: $\left\|\bar{x}_{0}^{\mathrm{ws}}-\bar{x}_{s}^{\tau, \text { tr }}\right\|_{P} \geq(1-\delta)\left\|\Delta \bar{x}_{s}^{\tau, \text { tr }}\right\|_{P}$.

Following similar arguments as in Case 1 it can be shown that $(1-\delta)\left\|\Delta \bar{x}_{s}^{\tau, \text { tr }}\right\|_{P} \leq\|A\|_{P}\left\|\Delta \bar{x}_{0}^{\tau, t r}\right\|_{2}+\|B\|_{P}\left\|\Delta \bar{u}_{0}^{\tau, t r}\right\|_{2}$ and by recalling that $\|\cdot\|_{Q}^{2} \leq\|\cdot\|_{P}^{2}$, there exists a constant $\bar{\epsilon}_{s} \in(0,1]$ such that $\bar{\epsilon}_{s}^{2}(1-\delta)^{2}\left\|\Delta \bar{x}_{s}^{\tau, \text { tr }}\right\|_{Q}^{2} \leq \bar{\epsilon}_{s}^{2}(1-\delta)^{2}\left\|\Delta \bar{x}_{s}^{\tau, t r}\right\|_{P}^{2} \leq\left\|\Delta \bar{x}_{0}^{\tau, \text { tr }}\right\|_{Q}^{2}+$ $\left\|\Delta \bar{u}_{0}^{\tau, t r}\right\|_{R}^{2}$. From this and (C.1a) we then obtain

$$
\begin{aligned}
\Delta V & \leq-\frac{1}{2}\left\|\Delta \bar{x}_{0}^{\tau, t r}\right\|_{Q}^{2}-\frac{1}{2} \bar{\epsilon}_{s}^{2}(1-\delta)^{2}\left\|\Delta \bar{x}_{s}^{\tau, t r}\right\|_{Q}^{2}-\left\|x^{-}-\bar{x}_{0}^{\tau, t r}\right\|_{Q}^{2} \\
& \leq-\frac{1}{4} \bar{\epsilon}_{s}^{2}(1-\delta)^{2}\left\|\bar{x}_{0}^{\tau, t r}-x_{r}\right\|_{Q}^{2}-\frac{1}{4}\left\|x^{-}-\bar{x}_{0}^{\tau, \text { tr }}\right\|_{Q}^{2} \\
& \leq-\frac{1}{8} \bar{\epsilon}_{s}^{2}(1-\delta)^{2}\left\|x^{-}-x_{r}\right\|_{Q}^{2}
\end{aligned}
$$

and the Lyapunov constraint is satisfied with $\epsilon_{k}=\frac{1}{4} \bar{\epsilon}_{s}^{2}(1-\delta)^{2}>0$. Case 2b: $\left\|\bar{x}_{0}^{\text {ws }}-\bar{x}_{s}^{\tau, \text { tr }}\right\|_{P}<(1-\delta)\left\|\Delta \bar{x}_{s}^{\tau, t r}\right\|_{P}$.

In this case it will be shown that $\alpha_{\min }<1$ by proving feasibility of the Lyapunov and terminal constraint in the minimization in Step 6 of Algorithm 2 for some $\alpha<1$. We make use of Lemma 5.6 in order to show that in Case $2 \mathrm{~b}$ the warm-start satisfies $V_{N}^{\text {tr }}\left(\overline{\mathbf{x}}^{\mathrm{ws}}\right.$, $\left.\overline{\mathbf{u}}^{\mathrm{ws}}, \bar{x}_{s}^{\mathrm{ws}}, \bar{x}_{s}^{\mathrm{ws}}\right) \leq V_{N}^{t r}\left(\overline{\mathbf{x}}^{\mathrm{ws}}, \overline{\mathbf{u}}^{\mathrm{ws}}, \bar{x}_{s}^{\tau, t r}, \bar{u}_{s}^{\tau, t r}\right)-(1-\delta)^{2}\left\|\Delta \bar{x}_{s}^{\tau, t r}\right\|_{P}^{2}$ and therefore from (C.1)

$$
\begin{aligned}
\Delta V & \leq-\left\|\Delta \bar{x}_{0}^{\tau, \text { tr }}\right\|_{Q}^{2}-\left\|x^{-}-\bar{x}_{0}^{\tau, \text { tr }}\right\|_{Q}^{2}-(1-\delta)^{2}\left\|\Delta \bar{x}_{s}^{\tau, \text { tr }}\right\|_{P}^{2} \\
& \leq-\frac{1}{2}\left\|x^{-}-\bar{x}_{s}^{\tau, t r}\right\|_{Q}^{2}-(1-\delta)^{2}\left\|\Delta \bar{x}_{s}^{\tau, t r}\right\|_{P}^{2} \\
& \leq-(1-\delta)^{2} \frac{1}{4}\left\|x^{-}-x_{r}\right\|_{Q}^{2} .
\end{aligned}
$$

The Lyapunov constraint is hence satisfied with $\epsilon_{k}=\frac{1}{2}(1-\delta)^{2}$ and $\alpha=\delta<1$ is feasible in the minimization in Step 6. Finally, feasibility with respect to the terminal constraint for some $\alpha<1$ remains to be shown. Recall that $\bar{x}_{N}^{\text {ws }}-\bar{x}_{s}^{\tau, t r}=A_{K}^{N}\left(\bar{x}_{0}^{\mathrm{ws}}-\bar{x}_{s}^{\tau, t r}\right)$ by using the auxiliary control law. We prove that $\left\|\bar{x}_{N}^{\text {ws }}-\bar{x}_{s}^{\text {ws }}\right\|_{P} \leq \epsilon_{s}$ for some $\alpha<1$, which implies that $\bar{x}_{N}^{\text {ws }} \in \bar{X}_{f}^{\text {tr }}\left(\bar{x}_{s}^{\text {ws }}, \bar{u}_{s}^{\text {ws }}\right)$ by the definition of $\epsilon_{s}$ :

$$
\begin{gathered}
\left\|\bar{x}_{N}^{\mathrm{ws}}-\bar{x}_{s}^{\mathrm{ws}}\right\|_{P}=\left\|\bar{x}_{N}^{\mathrm{ws}}-\bar{x}_{s}^{\tau, t r}+(1-\alpha)\left(\Delta \bar{x}_{s}^{\tau, t r}\right)\right\|_{P} \\
\leq\left\|A_{K}^{N}\left(\bar{x}_{0}^{\mathrm{ws}}-\bar{x}_{s}^{\tau, t r}\right)\right\|_{P}+(1-\alpha)\left\|\Delta \bar{x}_{s}^{\tau, t r}\right\|_{P} \leq \epsilon_{s},
\end{gathered}
$$

which is satisfied for some $\alpha<1$, since $\left\|A_{K}^{N}\left(\bar{x}_{0}^{\text {ws }}-\bar{x}_{s}^{\tau, \text { tr }}\right)\right\|_{P}<\epsilon_{s}$. As a result, there exists an $\alpha<1$ such that the terminal and Lyapunov constraint are feasible and $\alpha_{\min }<1$ will therefore be obtained in the minimization in Step 6, which concludes the proof.

\section{References}

Axehill, D., \& Hansson, A. (2008). A dual gradient projection quadratic programming algorithm tailored for model predictive control. In Proc. of the 47th IEEE conf. on decision and control. (pp. 3057-3064), 9-11 Dec.

Bemporad, A., \& Morari, M. (1999). Robust model predictive control: a survey. Robustness in Identification and Control, 245, 207-226.

Blanchini, F. (1999). Set invariance in control. Automatica, 35, 1747-1767.

Boyd, S., \& Vandenberghe, L. (2004). Convex optimization. Cambridge University Press.

Domahidi, A. (2012). Fast optimization for real-time control on embedded systems. http://forces.ethz.ch, Oct.

Domahidi, A., Zgraggen, A. U., Zeilinger, M. N., Morari, M., \& Jones, C. N. (2012). Efficient interior point methods for multistage problems arising in receding horizon control. In Proc. of the 51st IEEE conf. on decision and control. (pp. 668-674).

Ferramosca, A, Limon, D. Alvarado, I. Alamo, T. \& Camacho, E. F. (2009). MPC for tracking with optimal closed-loop performance. Automatica, 45(8), 1975-1978.

Ferreau, H. J., Bock, H. G., \& Diehl, M. (2008). An online active set strategy to overcome the limitations of explicit MPC. International Journal of Robust and Nonlinear Control, 18, 816-830.

Jiang, Z.-P., \& Wang, Y. (2001). Input-to-state stability for discrete-time systems. Automatica, 37, 857-869.

Lazar, M., \& Heemels, W. P. M. H. (2009). Predictive control of hybrid systems: inputto-state stability results for sub-optimal solutions. Automatica, 45, 180-185.

Lazar, M., Roset, B. J. P., Heemels, W. P. M. H., Nijmeijer, H., \& van den Bosch, P. P. J. (2008). Input-to-state stabilizing sub-optimal nonlinear MPC algorithms with an application to DC-DC converters. International Journal of Robust and Nonlinear Control, 18, 890-904.

Limon, D., Alamo, T., Raimondo, D. M., Muñoz de la Peña, D., Bravo, J. M., Ferramosca, A., \& Camacho, E. F. (2009). Input-to-state stability: a unifying framework for robust model predictive control. In Nonlinear model predictive control (pp. 1-26). Springer.

Limon, D., Alvarado, I., Alamo, T., \& Camacho, E. F. (2008). MPC for tracking piecewise constant references for constrained linear systems. Automatica, 44, 2382-2387.

Limon, D., Alvarado, I., Alamo, T., Fiacchini, M., \& Camacho, E. F. (2010). Robust tube-based MPC for tracking of constrained linear systems with additive disturbances. Journal of Process Control, 20(3), 248-260.

Löfberg, J. (2004) YALMIP: a toolbox for modeling and optimization in MATLAB. In Proc. of the CACSD Conference, Taipei, Taiwan.

Luenberger, D. G. (1984). Linear and nonlinear programming. Addison-Wesley.

Maciejowski, J. (2000). Predicive control with constraints. Prentice Hall.

Magni, L., Raimondo, D. M., \& Scattolini, R. (2006). Regional input-to-state stability for nonlinear model predictive control. IEEE Transactions on Automatic Control, 51(9).

Mayne, D. Q., Rawlings, J. B., Rao, C. V., \& Scokaert, P. O. M. (2000). Constrained model predictive control: Stability and optimality. Automatica, 36(6), 789-814.

Mayne, D. Q., Seron, M. M., \& Rakovic, S. V. (2005). Robust model predictive control of constrained linear systems with bounded disturbances. Automatica, $41,219-234$.

Milman, R., \& Davison, E. J. (2008). A fast MPC algorithm using nonfeasible active set methods. Journal of Optimization Theory and Applications, 139, 591-616.

Nocedal, J., \& Wright, S. J. (2006). Numerical optimization. In Springer series in operations research. New York: Springer.

Rao, C. V., Wright, S. J., \& Rawling, J. B. (1998). Application of interior-point methods to model predictive control. Journal of Optimization Theory and Applications, 99, 723-757.

Rawlings, J. B., \& Mayne, D. Q. (2009). Model predictive control: theory and design. Nob Hill Publishing.

Scokaert, P. O. M., Mayne, D. Q., \& Rawlings, J. B. (1999). Suboptimal model predictive control (feasibility implies stability). IEEE Transactions on Automatic Control, 44(3), 648-654. 


\section{ARTICLE IN PRESS}

Shahzad, A., \& Goulart, P. J. (2011). A new hot-start interior-point method for model predictive control. In Proc. of the 18th IFAC world congress. Milano.

Sontag, E. D., \& Wang, Y. (1999). New characterizations of input-to-state stability. IEEE Transactions on Automatic Control, 44, 648-654.

Toh, K. C., Todd, M. J., \& Tütüncü, R. H. (1999). SDPT3-A Matlab software package for semidefinite programming, Version 1.3. Optimization Methods $\mathcal{E}$ Software, 11(1-4), 545-581.

Vidyasagar, M. (1993). Nonlinear systems analysis (2nd ed.) Prentice Hall.

Wang, Y., \& Boyd, S. (2010). Fast model predictive control using online optimization. IEEE Transactions on Control Systems Technology, 18(2), 267-278.

Wright, S. J. (1997). Applying new optimization algorithms to model predictive control. In Chemical Process Control-V, CACHE: vol. 93 (pp. 147-155).

Zeilinger, M. N. (2011). Real-time model predictive control. Ph.D. thesis, ETH Zürich.

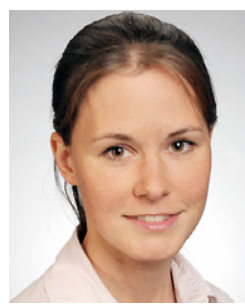

Melanie N. Zeilinger received the diploma in Engineering Cybernetics from the University of Stuttgart, Germany, in 2006. She conducted her diploma thesis research at the Department of Chemical Engineering, University of California at Santa Barbara, USA, in 2005-2006. In 2011 she received the Dr.sc. degree in Electrical Engineering from ETH Zurich, Switzerland. From 2011-2012 she was a postdoctoral fellow in the Automatic Control Laboratory at the École Polytechnique Fédérale de Lausanne (EPFL), Switzerland. She is currently a Marie Curie fellow at the Max-Planck Institute for Intelligent Systems, Tübingen, Germany, and a postdoctoral researcher at the University of California, Berkeley, USA. Her current research interests include real-time and distributed control and optimization and the development of modeling and control techniques for green energy-efficient technologies.

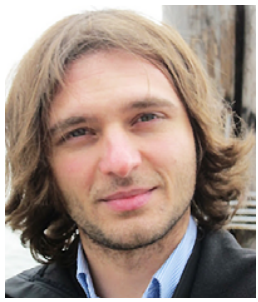

Davide M. Raimondo was born in Pavia, Italy, in 1981. He received the B.Sc. and M.Sc. in Computer Engineering and the Ph.D. in Electronic, Computer Science and Electric Engineering from the University of Pavia, Italy, in 2003, 2005, and 2009, respectively. As a Ph.D. student he held a visiting position at the Department of Automation and Systems Engineering, University of Seville, Spain. From January 2009 to December 2010 he was a postdoctoral fellow in the Automatic Control Laboratory, ETH Zürich, Switzerland. From March 2012 to June 2012 and from August 2013 to September 2013 he was a visiting scholar in Prof. Braatz Group, Department of Chemical Engineering, MIT, USA. Since December 2010 he is an Assistant Professor at University of Pavia, Italy. He is the author or coauthor of more than 50 papers published in refereed journals, edited books, and refereed conference proceedings. His current research interests include optimization-based control, fault-tolerant control, autonomous surveillance and control of glycemia in diabetic patients.

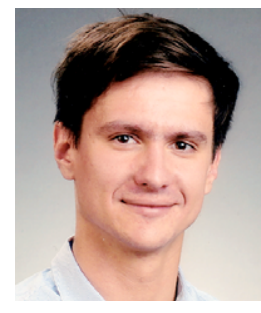

Alexander Domahidi received the Dipl.-Ing. degree in 2009 in electrical engineering and information technology from the RWTH University Aachen, Germany. He obtained the Dr.sc. degree from ETH Zurich, Switzerland in 2013 where he was with the Automatic Control Laboratory in 2009-2013. He has been an exchange student at Imperial College London, UK, in 2006-2007 and a scholarship holder of the German National Academic Foundation in 2005-2009. His current research interests are in real-time optimization methods for embedded applications. He is the main author of the two freely available interior point codes FORCES and ECOS, and is the co-founder of the Zurich based company embotech $\mathrm{GmbH}$, which focuses on tailored solutions for embedded optimization.

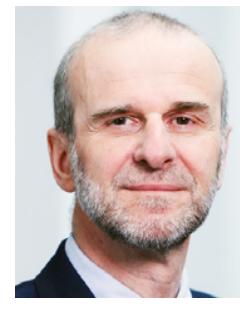

Manfred Morari was the Head of the Department of Information Technology and Electrical Engineering at ETH Zurich from 2009 to Jan 2012. He was the Head of the Automatic Control Laboratory from 1994 to 2008 Before that he was the McCollum-Corcoran Professor of Chemical Engineering and Executive Officer for Control and Dynamical Systems at the California Institute of Technology. He obtained the diploma from ETH Zurich and the Ph.D. from the University of Minnesota, both in chemical engineering. His interests are in hybrid systems and the control of biomedical systems. In recognition of his research contributions he received numerous awards, among them the Donald P. Eckman Award, the John R. Ragazzini Award and the Richard E. Bellman Contro Heritage Award of the American Automatic Control Council, the Allan P. Colburn Award and the Professional Progress Award of the AIChE, the Curtis W. McGraw Research Award of the ASEE, Doctor Honoris Causa from Babes-Bolyai University, Fellow of IEEE, IFAC and AIChE, the IEEE Control Systems Technical Field Award, and was elected to the National Academy of Engineering (U.S.). Manfred Morari has held appointments with Exxon and ICI plc and serves on the technical advisory boards of several major corporations.

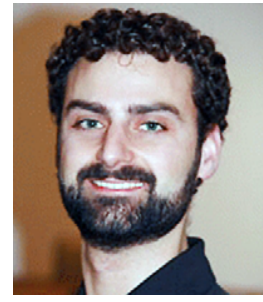

Colin N. Jones is an Assistant Professor in the Automatic Control Laboratory at the École Polytechnique Fédérale de Lausanne (EPFL) in Switzerland. He was a Senior Researcher at the Automatic Control Lab at ETH Zurich until 2010 and obtained a Ph.D. in 2005 from the University of Cambridge for his work on polyhedral computational methods for constrained control. Prior to that, he was at the University of British Columbia in Canada, where he took his bachelor and master degrees in Electrical Engineering and Mathematics. His current research interests are in the areas of high-speed predictive control and optimization, as well as green energy generation, distribution and management. 